\title{
Sistemas urbanos en América Latina: Roles y advenimientos de nuevos centros desde la perspectiva del transporte aéreo
}

\author{
Candida GAGO GARCÍA \\ Departamento de Análisis Geográfico Regional y Geografía Física (UCM). \\ cgago@ghis.ucm.es \\ Juan Antonio CóRDOBA ORDÓNEZ \\ Departamento de Análisis Geográfico Regional y Geografía Física (UCM). \\ jcordoba@ghis.ucm.es
}

Recibido: 18 de marzo de 2013

Enviado a evaluar: 15 de mayo de 2013

Aceptado: 8 de julio de 2013

\section{RESUMEN}

El trabajo presenta la situación actual del sistema urbano latinoamericano mediante el análisis de su evolución demográfica y utiliza el transporte aéreo como un indicador significativo de la vertebración y la cohesión regional. También se utilizan medidas de conectividad aérea para valorar la inserción de los nodos latinoamericanos en el escenario global. Mediante metodologías cuantitativas basadas en operaciones de estadística descriptiva se ponen de manifiesto los cambios y permanencias que se han producido en el sistema urbano de América Latina y del Caribe durante las últimas décadas, con objeto de identificar nuevas relevancias en el sistema urbano y la existencia de nodos que tienden a cumplir, cada vez más, una función global, actuando como centros de conexión necesaria entre esta región y el resto del mundo.

Palabras clave: Sistema urbano, América Latina y Caribe, globalización, transporte aéreo.

Urban system in Latin America:

Their roles and advent of new nodes from the air transportation perspective.

\begin{abstract}
The paper introduces the situation of Latin American urban system, analyzing the growth population, using primarily air transportation as an indicator of territorial structure and cohesion. Air transportation connectivity measures are recently used, as well, to assess the inclusion of the nodes on the global arena, as it is also the purpose of this study. Permanencies and changes in the Latin American and Caribbean urban system will be highlight using quantitative methodologies based on descriptive statistical operations. The aim of our work will be to identify those nodes that burst on the urban scene and those destined to play a global role as main linkages between the region and the world.
\end{abstract}

Key words: Urban system, Latin America and the Caribbean, globalisation, air transportation. 


\section{Rèseaux urbains en Amèrique Latine: Nouveaux enjeux dáprès la connexion aèrienne des villes.}

\section{RÉSUMÉ}

Ce travail présente l'actualité du réseau urbain latinoaméricain d'après l'analyse de la dinamique démographique récente et du transport aérien comme indicateur de l'articulation regionale. On s'aide de certaines mesures de liaisons aériennes à la fin de découvrir le branchement des nodes latinoaméricains dans un scénario mondial. D'après une méthodologie quantitative qui se sers de l'estatistique descriptive on essaie de relever des changements ou des permanences à l'intérieur des systèmes urbains lors des dernières décennies à l'object d'envisager de nouvelles significations pour les villes dans un contexte de mondialisation.

Mots clés: Événement, risque, brucellose bovine, comportement electoral, européisation.

\section{INTRODUCCIÓN}

Este trabajo pretende identificar la irrupción en el sistema urbano latinoamericano de núcleos con roles específicos al mismo tiempo que se realiza una valoración del sistema en relación con la conectividad aérea, tanto doméstica como internacional, concebida como un indicador de la funcionalidad de los nodos en los circuitos estatales, regionales y globales y del grado de integración regional y mundial. En este sentido, entendemos el transporte aéreo como una práctica que permite valorar los procesos de interconectividad que acontecen en el orden global. Los planteamientos iniciales parten de conceptos básicos en el análisis actual de los sistemas urbanos globales como es el de Ciudad Mundial y fundamentalmente de la constatación de que ésta constituye una tipología de nodos donde las consecuencias de la Globalización son máximas en términos de atracción y difusión de la movilidad. Este concepto servirá para valorar qué tipo de ciudades han irrumpido en el sistema y si están cumpliendo funciones globales, o por el contrario desempeñan otros roles de alcance nacional o regional.

Como premisa hay que señalar que un aspecto que se considera esencial en los procesos de Globalización actuales es la necesidad que tienen los territorios de estar altamente interconectados en la escala mundial a través una serie de ciudades con un valor estratégico de primer orden donde se localiza el poder económico al tiempo que son núcleos de concentración y producción de movilidad de todo tipo (ideas, transportes, capitales, personas, servicios...). Muchos autores identifican estas ciudades bajo el paraguas genérico de ciudad mundial o global. Para algunos de éstos la particularidad fundamental del momento reciente es que los flujos y los intercambios que se producen rompen la tradicional división entre Norte y Sur, estando las ciudades que se insertan en los procesos de globalización cada vez más desconectadas de sus Hinterland(er) nacionales o regionales (Sassen, 1991, 2001). Esta tesis sostiene que hay determinados lugares que pueden saltar su tradicional "fijación" a la trayectoria histórico-económica de los territorios en los que se insertan (regiones, países), para 
pasar a engrosar una élite territorial global que concentra actividades de todo tipo y que cada vez se desconecta más de sus entornos nacionales o regionales inmediatos.

Los trabajos sobre este tema han suscitado un importante debate dentro de las Ciencias Sociales, pues otros muchos autores señalan que la Globalización tiene efectos claramente disimétricos, y que si bien un grupo selecto de ciudades, a las que se han unido algunas pertenecientes a los países de economía emergente, concentran un poder de control y dirección, no ya solo económico, sino también geopolítico, cultural y simbólico, el contexto de división del trabajo continúa perpetuando la inequidad espacial y la concentración de los beneficios en un número limitado de centros (Friedmann 1986, 1995, Massey 1994, Taylor 1997; 2000, Derudder et Al., 2003). Respondiendo a estas dos perspectivas, el enfoque de Smith (2003) califica el concepto de ciudad mundial como reduccionista, señalando que las principales ciudades del planeta, más que centros de poder son "conmutadores", lugares donde se entrecruzan tiempos, espacios, experiencias y que no es posible ponerles límite ni fronteras. En este sentido, el presente trabajo se basa en una hipótesis que en parte suscribe y combina las tesis de Friedmann y de Smith, señalando que si bien en algunos casos las ciudades pueden adaptarse a los procesos globalizadores dando un salto cuantitativo y cualitativo en su proyección en el orden global, éste depende de aspectos fijados al territorio en un contexto de competitividad donde se aprovechan al máximo las ventajas comparativas (tecnología, formación y cualificación, salarios, derechos medioambientales, fiscales) y donde se mezclan dinámicas que actúan en diferentes dominios escalares.

Esta controversia académica resulta especialmente interesante en el estudio del sistema de ciudades latinoamericano y caribeño, teniendo en cuenta que se trata de un ámbito de la semiperiferia económica, pero que incluye importantes economías emergentes, como Brasil y México, además de algunas de las megalópolis más importantes y antiguas del planeta. El potencial demográfico, el capital humano, su posición en la semiperiferia económica auguraban para América Latina, al principio de la "era de la globalización" unas expectativas notables para que algunos de sus países se integraran dentro del concepto de Norte desarrollado, entonces en boga. Sin embargo, pese al crecimiento económico de los últimos años, la región continúa presentando una importante polarización económica y demográfica hacia América del Norte, que incluso se ha agudizado, por ejemplo, con una industria turística claramente dependiente. Durante el último cuarto del siglo XX los procesos de globalización incidieron en Latinoamérica de forma diferente a como lo habían hecho en los países del denominado "mundo desarrollado", y también en los NICs (Nuevos Países Industrializados) de la región Asia-Pacífico. La aceleración de estos procesos a escala mundial coincidió en Latinoamérica con una crisis económica y social de magnitudes insospechadas, en la que intervinieron fenómenos como la quiebra del modelo de industrialización por sustitución de importaciones y la crisis de la deuda externa. Los países latinoamericanos debieron realizar en los años 80-90's, durante la llamada "Década Perdida", serias reformas de reestructuración económica que han tenido un importante coste social, con aumento del desempleo industrial, precarización de las actividades productivas, aumento del subempleo y ocupación en actividades informales, además 
de un incremento general de las desigualdades socioeconómicas entre la población rica y pobre (PREALC, 1992; Gwynne, 1999; Dedecca y Baltar, 1999). Sin embargo hoy día, mientras que Europa y otras partes del mundo se hallan inmersas en una crisis económica de raíces financieras sin precedentes, América Latina y Caribe muestran unos índices de crecimiento considerables; la región experimentó un crecimiento medio del PIB del $6 \%$ en 2010 y aunque ha registrado una desaceleración como consecuencia de la crisis económica mundial, la tasa de crecimiento se situó en $4,3 \%$ y $3,2 \%$ en los años 2011 y 2012 respectivamente (CEPAL, 2012).

En este planteamiento inicial, por otro lado, se debe también valorar necesariamente el papel del transporte aéreo como un indicador de la afectación del sistema urbano latinoamericano por las dinámicas globales, al tiempo que puede poner en evidencia el papel adquirido por determinados lugares. Debe señalarse, ante todo, que el transporte aéreo proporciona a las ciudades condiciones óptimas de accesibilidad física, sobre todo en las relaciones de medio y largo alcance y, especialmente en la escala global. Esta aseveración es aún más relevante en los países donde las redes de transporte superficial son limitadas o inmaduras y en todos aquéllos países donde todavía no se han establecido redes ferroviarias de alta velocidad, como es el caso latinoamericano. Conviene también recordar que el transporte aéreo, como elemento y vehículo de la civilización neotécnica, ha intervenido sustancialmente en la reformulación de conceptos espaciales debido a la movilidad y accesibilidad, afectando cada vez a segmentos más amplios de la población, sean políticos, empresarios, empleados, cooperantes, estudiantes, turistas o emigrantes (Córdoba et Al. 2007).

Además, desde los años 70's del siglo pasado, el transporte aéreo ha perdido paulatinamente su condición de transporte elitista. Su popularización creciente ha estado relacionada con varios hitos en la aviación comercial, entre ellos, la generalización de los aviones de gran capacidad, la difusión de los vuelos charter, de forma mucho más reciente la proliferación de las aerolíneas de bajo coste $\mathrm{y}$, en términos generales, la creciente liberalización de la aviación comercial en muchos países. Esta tesis permite sostener que, actualmente, el transporte aéreo no solo potencia la accesibilidad física de los lugares sino también la accesibilidad en términos económicos e incluso sociales. En esta misma línea se puede argumentar que el transporte aéreo, al facilitar la accesibilidad, contribuye a mejorar la condiciones de movilidad, una tesis que concierne a los principales elementos implicados en los procesos de globalización: personas (sean locales o foráneos, turistas o trabajadores, migrantes en cualquier caso), bienes e incluso capitales e ideas.

Por otra parte, desde un punto de vista metodológico, el transporte aéreo ha resultado ser un indicador muy valioso a la hora de valorar la centralidad y organización de los sistemas urbanos, y se ha utilizado tradicionalmente para determinar intensidades de relación entre pares de ciudades a partir de las cuales se han realizado, posteriormente, definiciones de jerarquías (Smith y Timberlake, 2001; Gago, 2003; Derudder y Wiltox 2005). En cualquier caso, actualmente es inconcebible que una ciudad que pretenda participar en el sistema global de relaciones pueda estar lejos de un aeropuerto. Tampoco parece sensato, de cara a unas condiciones de competitividad 
adecuadas en el escenario global, que una ciudad con un volumen elevado de población esté desprovista de relaciones aéreas que la conecten con otros sistemas regionales e internacionales. Finalmente, además de la relevancia que pueda tener el análisis del transporte aéreo para el conocimiento de la estructura y jerarquías urbanas, su estudio es conveniente para el conocimiento de la realidad social y territorial contemporánea.

Los contenidos de este artículo relacionan, por lo tanto, los conceptos de movilidad, transporte aéreo, jerarquía urbana e inserción de las ciudades en el orden global, organizándose en cuatro apartados más. En primer lugar se exploran los cambios recientes en el sistema urbano latinoamericano a partir de una valoración del crecimiento demográfico como primer paso para identificar aquellos nodos con un dinamismo creciente, haciéndose también una presentación de los roles que cumplen éstos últimos. El siguiente apartado se centra en la identificación de aquellos núcleos que tienen una conectividad diferencial y valora la interconexión que proporciona al territorio la red doméstica de transporte aéreo como un indicador de la cohesión territorial. Después se evalúa la inserción de las ciudades en los circuitos globales mediante el análisis de su conectividad internacional. Por último se presentarán las principales conclusiones y las líneas de debate que se abren a partir de las mismas.

\section{TRANSFORMACIONES DE LOS SISTEMAS URBANOS DE AMÉRICA LATINA Y CARIBE EN LAS DOS ÚLTIMAS DÉCADAS}

El último informe de ONU-Habitat señalaba que, en 2010, con un valor del $80 \%$ América Latina había alcanzado la tasa de urbanización más elevada del planeta (ONU-Habitat, 2012). De hecho, algunos países latinoamericanos tienen algunas de las tasas estimadas más elevadas del mundo en 2011: 93,5\% en Venezuela; 92,58 \% en Uruguay; $92,58 \%$ en Argentina y $92,56 \%$ en Chile, valores que aún se superan en territorios insulares como Guadalupe $(98,4 \%)$ y Puerto Rico $(98,9 \%)$ y que llegan a ser notables en países de grandes dimensiones como Brasil $(86,9 \%)$ o México $(77,8 \%)$ (ONU, 2010, INEGI, para México ${ }^{1}$ ). También es conocido el efecto polarizador que ejercen las ciudades latinoamericanas sobre sus regiones debido en gran parte a tres particularidades: la concentración geográfica del fenómeno urbano, la inexistencia, en muchos países, de verdaderos sistemas urbanos y la inmadurez, cuando existen,

\footnotetext{
${ }^{1}$ Es prácticamente imposible lograr series estadísticas cronológicamente homogéneas para la población urbana latinoamericana. Nuestras fuentes de detalle han sido los diferentes censos elaborados por las oficinas oficiales de estadística de cada país. Para datos generales hemos utilizado las cifras que han propuesto el Banco Mundial en sus informes sobre el Desarrollo Mundial y el PNUD en los Informes de Desarrollo Humano. En ciertos casos puntuales ha sido necesario acudir a fuentes locales (siempre oficiales). Ha sido de gran ayuda la World Gazetteer (http://world-gazetteer.com/) para el establecimiento inicial de hipótesis, aunque sus datos han debido ser contrastados con fuentes oficiales, particularmente en el caso de la delimitación de áreas metropolitanas.
} 
porque están caracterizados a menudo por la macrocefalia y/o por el centralismo acusado de algunas ciudades (Díaz Orueta y Lungo, 1999; Pinto da Cunha, 2003).

En las últimas décadas, sin embargo, se están produciendo cambios interesantes en las tendencias de los procesos de urbanización latinoamericana (Aguilar y Rodríguez, 1995; Aguilar y Vázquez 2000; Sassone, 2000; Sánchez-Crispín y Propín, 2001; Ortiz et Al., 2003; Ariza, M. 2003. Córdoba y Gago, 2010). Debe señalarse, ante todo, que el crecimiento de la población urbana se ha ralentizado en la mayoría de los países a tenor de una ralentización general del crecimiento demográfico $(2,42 \%$ anual en 1970-80 frente a 1,43\% en 2000-05), un fenómeno que está íntimamente relacionado con la fuerte caída de las tasas de fecundidad (5\% en $1970-75$ y $2,5 \%$ en $2000-05$ ) pero también con una importante sangría migratoria hacia Estados Unidos, Canadá y Europa. En términos generales se ha observado también que desde los años ochenta las mayores tasas de crecimiento urbano se han desplazado desde las grandes metrópolis hacia ciudades de tamaño intermedio; de hecho ya la mitad de la población urbana latinoamericana reside en ciudades de menos de 500.000 habitantes (ONUHábitat, 2012).

La población urbana latinoamericana ha registrado, no obstante, comportamientos diferenciales entre países y entre las propias regiones de un mismo país. Dos de los elementos más relevantes de este comportamiento diferencial han sido (Cuadro 1):

1.- Unas tasas medias anuales de crecimiento de la población urbana para 19952010 modestas en países como Argentina, Chile y Uruguay que ya en los años setenta tenían un nivel de urbanización considerable.

2.- Unas tasas medias anuales de crecimiento de la población urbana para 19952010 elevadas en países que en los años setenta tenían niveles medios o modestos de urbanización como Haití, Honduras, Paraguay, El Salvador, República Dominicana, Ecuador o Costa Rica, casi todos ellos con un peso considerable de las actividades primarias tanto en el empleo como en la formación del PIB y algunos de ellos con un componente demográfico amerindio muy fuerte, como Guatemala, Bolivia y El Salvador. En estos países estamos evidentemente en presencia de un éxodo rural relativamente tardío para el contexto latinoamericano.

El crecimiento desorbitado de algunas ciudades a expensas del medio rural se reconoce también en centros de regiones depauperadas cuyos datos quedan enmascarados por las medias estadísticas (cuadro 2); en muchos casos se trata del subdesarrollo dentro del subdesarrollo (Sur de México, regiones andinas o amazónicas) que afecta sobre todo, esto es sabido, a las condiciones de marginación extrema en que viven muchos grupos amerindios y afrodescendientes incluso en el seno de países con niveles de bienestar que empiezan a ser aceptables. El crecimiento diferencial y detallado de las ciudades latinoamericanas es, sin embargo, un fenómeno demasiado 
complejo que desborda el marco de este trabajo y que ya ha sido tratado por otras investigaciones ${ }^{2}$. Tan sólo podemos apuntar aquí algunas directrices relevantes:

1.- El crecimiento "moderado" de las ciudades muy grandes e incluso de sus periferias metropolitanas. Así se han comportado desde hace más una década aglomeraciones como Ciudad de México (1,42\% en 1995-05), Bogotá (1,75\% para 1995-05), Sao Paulo y Río de Janeiro (1,67\% y 1,07\% para 1996-05), Buenos Aires (1,56\% para 1990-00) o Santiago de Chile (1,07\% para 1992-02). Por emulación, otras grandes ciudades han seguido también estas pautas de crecimiento moderado, como Guadalajara y Monterrey en México con $1,27 \%$ y $1,82 \%$; Medellín y Cali en Colombia con $1,67 \%$ y $1,42 \%$; Porto Alegre y Recife en Brasil con $1,56 \%$ y $1,51 \%{ }^{3}$. Esta contención, mucho más acusada en el periodo actual, es la respuesta a la ralentización general del crecimiento demográfico, pero se trata también de una contención en los flujos migratorios que se desvían hacia núcleos emergentes en los propios países o hacia el exterior. Sospechamos, que en algunos casos se trata más que de una verdadera contención, de un fenómeno de metropolitanización de escala inusual y asociada a conceptos como los de ciudad-región o ciudad-difusa: a veces el crecimiento de estas grandes ciudades no se da ya en sus propias áreas metropolitanas ni en sus ciudades satélite (Puebla o Toluca para Ciudad de México, por ejemplo con tasas de $1,90 \%$ y 3,18\%) sino en las nuevas áreas metropolitanas de éstas últimas (San Bernardino Tlaxcalancingo (Cholula) 19\%, o Amozoc de Mota, 6,22\%, para Puebla, Ojo de Agua, 22\% para Pachuca, o Zinantepec 2,72\% para Toluca) ${ }^{4}$. Se estarían conformado así, en las últimas décadas, verdaderas regiones metropolitanas polinucleares (Garza 2000; Chion, 2002; Aguilar, 2002; Aguilar \& Ward 2003; ONUHábitat 2010), que albergan a más de 30 millones de habitantes en áreas con radios superiores a los $100 \mathrm{Km}$. lineales en casos como los de Ciudad de México o Sao Paulo o con valores inferiores pero también extraordinarios en casos como Santiago de Chile, Buenos Aires, Lima o Caracas.

2.- En un segundo nivel de diferenciación en cuanto a las tasas de crecimiento deben considerarse las ciudades grandes y medias de los diferentes sistemas urbanos nacionales. Como tónica general, estas ciudades han mantenido un ritmo de crecimiento superior al de las ciudades primadas de cada sistema, sobre todo teniendo en cuenta que están en proceso de articular sus propias áreas metropolitanas: Ciudad Juárez (1,12\%), Tijuana (1,32\%), León (2,13\%), San Luis Potosí (1,48 \%), Querétaro (1,67\%) en México; Rosario (1,65\%), Salta (1,58\%) o Córdoba (0,5\%) en Argentina; Manaus (2,85\%), Brasilia (2,2\%), Aracaju $(1,83 \%)$, João Pessoa $(1,68)$, Goiania $(1,64)$ en Brasil; Barinas (3,44\%), Maturín (3,4\%), Turmero (3,1\%), Maracaibo $(2,12 \%)$, Cumaná $(1,77 \%)$, Valencia $(1,18 \%)$ en Venezuela. Todas son ciudades han manteni-

\footnotetext{
${ }^{2}$ Ver autores antecitados, en este mismo epígrafe.

${ }^{3}$ Crecimiento medio referido a las mismas fechas que las capitales estatales, citadas arriba.

${ }^{4}$ Crecimiento anual medio para el periodo 2000-2010. Fuente, INEGI.
} 
do entre 2000 y 2010 aproximadamente tasas de crecimiento considerablemente superiores a otras de las ciudades principales y nos informan sobre un proceso, al menos incipiente, de rearticulación regional en los respectivos países.

Cuadro 1. Tendencias de la población urbana en algunos países de Latinoamérica.

\begin{tabular}{|c|c|c|c|c|c|c|c|}
\hline & \multirow{2}{*}{\multicolumn{4}{|c|}{$\begin{array}{c}\text { Población urbana } \\
\%\end{array}$}} & \multicolumn{3}{|c|}{ Tasa crecimiento anual medio } \\
\hline & & & & & $\%$ & $\%$ & $\%$ \\
\hline & 1970 & 1990 & 2005 & 2011 & $1995-2000$ & 2000-2005 & 2005-2010 \\
\hline Costa Rica & 38,8 & 47,0 & 61,7 & 64,9 & 1,13 & 0,88 & 0,79 \\
\hline El Salvador & 39,0 & 44,0 & 59,8 & 64,8 & 1,75 & 0,91 & 0,84 \\
\hline Paraguay & 37,1 & 48,0 & 58,5 & 62,08 & 1,19 & 1,10 & 0,97 \\
\hline Bolivia & 36,2 & 51,0 & 64,2 & 67,00 & 0,80 & 0,75 & 0,68 \\
\hline Guatemala & 36,2 & 39,0 & 47,2 & 49,94 & 0,91 & 0,89 & 0,89 \\
\hline Haití & 19,7 & 28,0 & 38,8 & 53,55 & 1,77 & 4,27 & 3,30 \\
\hline Honduras & 29,0 & 44,0 & 46,5 & 52,2 & 1,14 & 1,36 & 1,17 \\
\hline Rep. Domin. & & 55 & 66 & 70 & 2,74 & 2,39 & 2,03 \\
\hline Ecuador & & 55 & 64 & 67 & 2,48 & 2,75 & 2,49 \\
\hline Chile & 73,0 & 86,0 & 87,6 & 89,2 & 0,37 & 0,39 & 0,30 \\
\hline Argentina & 78,4 & 86,0 & 90,1 & 92,56 & 0,33 & 0,28 & 0,21 \\
\hline Uruguay & 82,0 & 86,0 & 92,0 & 92,58 & 0,17 & 0,13 & 0,11 \\
\hline
\end{tabular}

Fuentes: THE WORLD BANK, Stimations to World Development Reports. ONU, World Population Prospects, 2010, The Revision, Nueva York. ONU. Tabla: Average annual rate of change of the percentage urban, 1950-2050.

3.- Un tercer nivel de diferenciación nos lleva a considerar el crecimiento de ciudades de diferente peso demográfico pero animadas por un dinamismo especial y a veces espectacular que obliga a recurrir al viejo concepto de ciudades hongo. En este grupo, muy numeroso, tienen cabida (véase el cuadro 3 ): 
Cuadro 2. Tendencias de la población urbana en algunos países y ciudades de Latinoamérica.

\begin{tabular}{|c|c|c|c|c|c|c|}
\hline País & $\%$ & Ciudad & $\%$ & Año & $\%$ & Año \\
\hline México & & Oaxaca (Oaxaca) & 7,14 & \multirow{4}{*}{$1990-2000$} & & \\
\hline $1990-2000$ & 2,4 & $\begin{array}{l}\text { S. Cristóbal de las Casas } \\
\text { (Chiapas) }\end{array}$ & 5,41 & & 4,05 & \multirow{3}{*}{$2000-2010$} \\
\hline \multirow[t]{2}{*}{$2000-2010$} & \multirow[t]{2}{*}{1,8} & Tuxtla Gutiérrez (Chiapas) & 4,69 & & 2,66 & \\
\hline & & $\begin{array}{c}\text { Comitán de Domínguez } \\
\text { (Chiapas) }\end{array}$ & 4,64 & & 3,87 & \\
\hline Perú & & $\begin{array}{l}\text { Puerto Maldonado (Madre } \\
\text { de Dios) }\end{array}$ & 6,5 & \multirow{7}{*}{$1993-2007$} & 2 & \multirow{7}{*}{$\begin{array}{l}\text { 2007-2012. } \\
\text { (Proy.) }\end{array}$} \\
\hline \multirow[t]{6}{*}{$(1993-2007)$} & \multirow[t]{6}{*}{2,4} & Sullana (Piura) & 5,7 & & 4,4 & \\
\hline & & Paita (Piura) & 5,2 & & 4,2 & \\
\hline & & $\begin{array}{c}\text { Ayacucho (Ayacucho, } \\
\text { Sierra) }\end{array}$ & 4,37 & & 3,37 & \\
\hline & & Juliaca (Puno) & 3,85 & & 2,98 & \\
\hline & & $\begin{array}{c}\text { Pucallpa (Coronel Portillo, } \\
\text { Ucayali) }\end{array}$ & 3,7 & & 2,64 & \\
\hline & & Tarapoto (Dep. San Martín) & 3,2 & & 12,1 & \\
\hline Colombia & & Apartadó (Antioquia) & 13,7 & \multirow{5}{*}{$1985-2005$} & 5,1 & \multirow{5}{*}{$\begin{array}{c}2005-2012 \\
\text { Proy. del } \\
\text { DANE }\end{array}$} \\
\hline \multirow[t]{2}{*}{$1990-2000$} & \multirow[t]{2}{*}{2,6} & Yopal (Casanare) & & & 5,05 & \\
\hline & & Quibdó (Chocó) & 5,05 & & 3,56 & \\
\hline \multirow[t]{2}{*}{$2000-2010$} & \multirow[t]{2}{*}{2,1} & Villavicencio (Meta) & 4,97 & & 3,11 & \\
\hline & & Valledupar (César) & 4,9 & & 2,81 & \\
\hline Ecuador & & Daule (Guayas) & & & 10,5 & \multirow{5}{*}{$2000-2010$} \\
\hline \multirow[t]{2}{*}{$1990-2000$} & \multirow[t]{2}{*}{3,1} & Esmeraldas (Esmeraldas) & & & 6,1 & \\
\hline & & Loja (Loja) & & & 4,3 & \\
\hline \multirow[t]{2}{*}{$2000-2010$} & \multirow[t]{2}{*}{2,9} & Nueva Loja (Sucumbíos) & & & 4,07 & \\
\hline & & Cuenca (Azuai) & & & 3,3 & \\
\hline
\end{tabular}

Fuente: Cálculo propio a partir de la población de las ciudades. Oficinas Nacionales de Estadística de los diferentes países, según las fechas de los diferentes censos u otras estadísticas. Los datos medios para México, Colombia y Ecuador pertenecen al Banco Mundial. 
Cuadro 3. Algunas ciudades de crecimiento acelerado en Latinoamérica.

\begin{tabular}{|c|c|c|c|c|c|c|c|}
\hline \multicolumn{8}{|c|}{ Ciudades seleccionadas. Tasa anual de crecimiento } \\
\hline País & Ciudad & $\%$ & Año & País & Ciudad & $\%$ & Año \\
\hline \multicolumn{4}{|c|}{ Éxodo rural retardado } & \multicolumn{4}{|c|}{ Desarrollos turísticos } \\
\hline Guatemala & Chchicastenango & 22,35 & 02-08 & México & Playa Carmen & 24,30 & $00-10$ \\
\hline Guatemala & Totonicapan & 16,92 & $02-08$ & México & S. José Cabo & 12,43 & $00-10$ \\
\hline Guatemala & Escuintla & 6,67 & 02-08 & México & Pto. Peñasco & 8,62 & $00-10$ \\
\hline Guatemala & C. de Guatemala & 6,03 & 02-08 & México & Cancún & 5,81 & $01-10$ \\
\hline Guatemala & Puerto Barrios & 5,89 & 02-08 & Costa Rica & Liberia & 5,37 & $00-10$ \\
\hline Honduras & San Pedro Sula & 4,41 & 01-05 & Brasil & Florianópolis & 2,59 & $00-10$ \\
\hline Honduras & Tegucigalpa & 4,21 & 01-05 & Brasil & João Pessoa & 2,11 & $00-10$ \\
\hline Haití & Gonaïves & 8,07 & $92-05$ & Dom. Rep. & Higüey & 13,55 & 02-08 \\
\hline Haití & Port-au-Prince & 5,46 & $92-05$ & Dom. Rep. & S. P. Macoris & 9,15 & 02-08 \\
\hline Bolivia & Santa Cruz & 5,01 & $01-10$ & Argentina & El Calafate & 18,3 & 00-09 \\
\hline Bolivia & Sucre & 5,16 & $01-10$ & \multicolumn{4}{|c|}{ Ciudades del petróleo } \\
\hline Bolivia & $\begin{array}{c}\text { Cochabamba } \\
\text { (aglm.) }\end{array}$ & 3,92 & $01-10$ & Venezuela & Maturín & 3,40 & $01-11$ \\
\hline \multicolumn{4}{|c|}{ Ciudades-frontera } & Venezuela & El Tigre & 2,34 & $01-11$ \\
\hline México & Reynosa & 4,6 & $00-10$ & Venezuela & Anaco & 1,12 & $01-11$ \\
\hline México & Nogales & 3,54 & $00-10$ & México & C. del Carmen & 3,44 & $00-10$ \\
\hline México & Agua Prieta & 2,78 & $00-10$ & \multicolumn{4}{|c|}{ Ciudades de colonización } \\
\hline México & Mexicali & 2,54 & $00-10$ & Brasil & Paraupebas & 13,44 & $00-10$ \\
\hline México & Nuevo Laredo & 2,1 & $00-10$ & Brasil & Palmas & 6,60 & $00-10$ \\
\hline Argentina & Ushuaia & 5,80 & $01-08$ & Brasil & Porto Velho & 4,28 & $00-10$ \\
\hline Paraguay & Ciudad del Este & 6,13 & $02-08$ & Brasil & Boa Vista & 4,1 & $00-10$ \\
\hline Bolivia & Yacuiba & 8,16 & $01-10$ & Brasil & Marabá & 3,87 & $00-10$ \\
\hline Bolivia & Tarija & 4,78 & $01-10$ & Brasil & Rio Branco & 3,64 & $00-10$ \\
\hline
\end{tabular}

Fuente: Oficinas Nacionales de Estadística de los diferentes países. 
1.- Muchas ciudades y "nuevas ciudades" de los países que están conociendo un éxodo rural acelerado en el último decenio, particularmente en Guatemala (con los casos extremos de Chichicastenango y Totonicapán), Honduras, Haití ${ }^{5}$ y Bolivia.

2.- Ciudades con una localización estratégica y, especialmente, las fronterizas, sobre todo en México pero también en el Cono Sur y en otras localizaciones.

3.- Ciudades cuyas tasas de crecimiento están indudablemente potenciadas por la puesta en valor de unos recursos que están atrayendo fuertes contingentes migratorios. Son a veces crecimientos explosivos que informan sobre la precariedad en la que se deben estar produciendo los nuevos asentamientos. En este grupo se integran muchas "ciudades del petróleo" de Venezuela; ciudades en frentes de colonización agropecuaria o forestal particularmente en Brasil (Godfrey y Browder, 1996), Perú, incluso Ecuador (Ryder \& Brown, 2000), y sobre todo, no tanto los centros turísticos cuanto las ciudades que viven de ellos: Playa del Carmen en la Riviera Maya de México y Higüey, próximo a Punta Cana y a La Romana, en República Dominicana son casos sobresalientes.

Este análisis, forzosamente sucinto de las tendencias generales de la urbanización latinoamericana nos induce a plantear que la moderación estadística general en las tasas de crecimiento de la población en general como de la población específicamente urbana enmascara importantes individualismos locales y regionales dignos de atención. Pensamos que se están produciendo ajustes significativos en los sistemas urbanos, unos sistemas que generalmente han sido definidos como inmaduros o simplemente inexistentes. Estas tendencias, que ya han sido estudiadas en detalle en casos como el de Brasil (Fernandes y Negreiros, 2001; Mata et Al., 2007) o México (Gutiérrez de McGregor y González, 2004) deben estar sin duda relacionadas con mecanismos de ajuste en el interior de los propios sistemas nacionales (saturación de mercados laborales, apertura de nuevos mercados en relación con la puesta en valor de nuevos territorios o recursos, decrecimiento de las oportunidades económicas en los medios rurales, redistribución del potencial del mercado de productos y servicios), pero también deberían responder a una nueva lógica global que desborda los ámbitos nacionales. ¿Hasta qué punto es correcta o se cumple esta hipótesis? ¿Siguen siendo las ciudades latinoamericanas meras aglomeraciones de "slums" o empiezan, al menos algunas, a descollar en el escenario internacional de competencias?

\footnotetext{
${ }^{5}$ La tendencia en las tasas de urbanización de Haití puede considerarse válida hasta el Terremoto de 2010.
} 


\section{TRANSPORTE AÉREO Y SISTEMAS URBANOS ESTABLES}

Esta sección se centra en el análisis de diferentes indicadores de conectividad aérea general y de conectividad en el interior de los diferentes estados con el objeto de ser interpretados como indicios de interacción y cohesión territorial, respondiendo en parte a los interrogantes formulados en el epígrafe anterior. Paralelamente, estos indicadores se relacionarán con parámetros de orden demográfico lo que, como veremos, permite identificar el comportamiento diferencial de algunos núcleos y valorar la magnitud de su integración en el sistema urbano. Con estos objetivos y desde un punto de vista metodológico, se ha elaborado una base de datos, manejando información de 354 ciudades con servicios aéreos domésticos o internacionales, reuniendo datos de tráfico de pasajeros totales y de volumen demográfico, todo ello referido al año 2010, lo que sin duda mejora de manera extraordinaria la comparación de la información ${ }^{6}$. A partir de estos datos se han aplicado medidas de estadística descriptiva que permiten su mejor interpretación.

Una primera aproximación al transporte aéreo latinoamericano nos habla de su escasa dimensión en el concierto mundial. A pesar de que existen en Latinoamérica algunas de las principales megalópolis del mundo y numerosos aglomerados urbanos con más de 1 millón de habitantes, ningún aeropuerto de la región se encuadra entre los 20 más importantes del mundo. El aeropuerto latinoamericano con mayor tráfico aéreo de pasajeros, el de Ciudad de México, apenas mueve la tercera parte del tráfico de Atlanta en EE.UU, a lo que se debe sumar, como veremos en el epígrafe 4, una importante polarización de las frecuencias por los Estados Unidos, lo que le resta al mismo relevancia en el escenario global. Los dos grandes aeropuertos de Sao Paulo, una aglomeración con más de 20 millones de personas, apenas mueven la misma cantidad de pasajeros que una ciudad europea como Munich $^{7}$ (cuadro 4).

Un simple análisis de dispersión revela, casi de inmediato, una relación lógica entre la población de la ciudad y el volumen de pasajeros que usan el transporte aéreo, pero esta correlación, en general positiva, debe analizarse con precaución deteniéndose cuidadosamente en las excepcionalidades en cada uno de los países (cuadro 5). Debe señalarse la deficiente correlación que existe si en vez de utilizar valores absolutos otorgamos un rango jerárquico (coeficiente de correlación de Spearman) tanto al

\footnotetext{
${ }^{6}$ Las fuentes sobre tráfico aéreo y volumen de pasajeros utilizadas en la presente investigación son las siguientes:

- Los datos de tráfico aéreo se han obtenido de Airports Council Internacional, pero se han tenido con completar de manera significativa con diferentes organismos oficiales de cada país.

- Para el análisis de conectividad aérea entre pares de ciudades, tanto de tráfico doméstico como internacional se han utilizado las Guías ABC para los años 1970, 1996 y 2011; año 1970: Thos Skiner and Co. (1970): ABC World Airways Guide, October 1970. Londres, Thos Skinner And Co.; años 1996 y 2011: bases de datos adquiridas a Comber International Guides - OAG, (frecuencias de vuelos nacionales y domésticos en los aeropuertos de los países del mundo; vuelos regulares).

${ }_{7}$ Aeropuerto de Munich: pasajeros totales, 37,8 millones en 2011. Fuente: Web del aeropuerto, http://www.munich-airport.de/en/micro/20jahre/statistik/index.jsp
} 
volumen demográfico como al total de pasajeros que utilizan los aeropuertos. Con este segundo grupo de valores se obtienen cifras intermedias para el conjunto latinoamericano $(0,58)$, aunque éstas se alejan mucho de valores aceptables en términos de correspondencia en algunos países -México, Chile, Ecuador- llegando a ser negativa en los casos de Argentina y Perú.

Este hecho no es nuevo en transporte aéreo porque se sabe que el tráfico de un aeropuerto responde a menudo a factores más complejos que el simple volumen de población de la ciudad o de la región a las que sirve; intervienen en esta correlación, además, las condiciones de competitividad de los transportes superficiales, una determinada opción en la política de transportes, que puede decidir primar una infraestructura en detrimento de otra, y otros muchos factores como el propio dinamismo y/o especialización funcional de los centros según su capacidad de generar demanda de transporte aéreo, o la falta de accesibilidad terrestre de determinadas localizaciones por ubicarse en medios naturales de accesibilidad complicada (selvas, topografías abruptas, áreas pantanosas, enclaves insulares).

Ante todo, es necesario resaltar que el flujo aéreo en Latinoamérica está generado por factores más complejos que el potencial demográfico de sus ciudades y la atracción que éstas puedan ejercer entre sí, entendidos dentro de parámetros puramente gravitatorios. En este sentido, los gráficos de dispersión para la correlación tráfico aéreo/población del núcleo urbano ${ }^{8}$, muestran algunos hechos significativos (fig. 1 y 2):

1.- El papel destacado de los aeropuertos que sirven a centros turísticos: Cancún, Punta Cana, Montego Bay, Foz de Iguaçu, Porlamar, Puerto Vallarta, Los Cabos son ejemplos destacados;

2.- El papel igualmente destacado de algunas capitales nacionales en donde el transporte aéreo alcanza un papel superior al que corresponde a su volumen poblacional (Brasilia, Caracas y San Juan) y de otras en donde se observa una correspondencia entre rango demográfico y aeroportuario, tales como Bogotá, La Habana, Lima y Santiago de Chile.

3.- Capitales con una conectividad aérea no ajustada a su rango demográfico como Tegucigalpa, La Paz o Asunción.

4.- La dualidad en la relevancia de los aeropuertos regionales dependiendo de los países; encontramos así aeropuertos como San Luis de Potosí, Saltillo, Querétaro, Xalapa en México un protagonismo escaso frente a otros como Guadalajara y Monterrey donde el volumen de pasajeros se ajusta al rango demográfico. Este desajuste es especialmente importante en los casos de Argentina (Rosario), Chile (Concepción) y Perú, pero también en algunos aeropuertos de Brasil, aunque en este caso debe indicarse que el sistema parece ajustado en los nodos superiores de la jerarquía urbana

\footnotetext{
${ }^{8}$ El potencial demográfico de los aeropuertos se ha definido a partir de la definición oficial de área metropolitana en cada ciudad.
} 
(Portoalegre, Salvador, Recife, Fortaleza y Curitiba). En este sentido se observan también sistemas bastante ajustados en la cúspide de la jerarquía urbana en los casos de Colombia y Ecuador.

5.- Por último, el protagonismo que ostentan las islas del Caribe (con San Juan de Puerto Rico a la cabeza) en el tráfico aéreo de la región, como consecuencia de la necesidad de desenclave aéreo, pero también debido a su creciente función turística y a su condición, en ciertos casos, de paraísos fiscales, aspectos que sospechamos pueden desempeñar un papel fundamental en su conectividad, aunque no pueda demostrarse con métodos convencionales de análisis.

Cuadro 4. Datos comparativos de los principales aeropuertos del mundo y de Latinoamérica. 2011.

\begin{tabular}{|r|c|c|c|c|}
\hline \multicolumn{5}{|c|}{ Aeropuertos con más tráfico de pasajeros entre los cien primeros del mundo. } \\
\hline Puesto & País & código IATA & Ciudad/aeropuerto & Pasajeros \\
\hline 1 & EE.UU. & ATL & Atlanta/Hartsfield-Jackson & 92.365 .860 \\
\hline 2 & China & PEK & Beijing/Capital International & 77.403 .668 \\
\hline 3 & Reino Unido & LHR & London/Heathrow & 69.433 .565 \\
\hline 4 & EE.UU. & ORD & Chicago/O'Hare & 66.561 .023 \\
\hline 5 & Japón & HND & Tokyo/International & 62.263 .025 \\
\hline 6 & EE.UU. & LAX & Los Angeles/International & 61.848 .449 \\
\hline 7 & Francia & CDG & Paris/Charles de Gaulle & 60.970 .551 \\
\hline 8 & EE.UU. & DFW & Dallas/Dallas-Forth Worth & 57.806 .152 \\
\hline 9 & Alemania & FRA & Frankfurt & 56.436 .255 \\
\hline 10 & China & HKG & Hong Kong & 53.314 .213 \\
\hline 45 & BEROPUERTOS CON MÁS PASAJEROS EN LATINOAMÉRICA & 30.371 .131 \\
\hline 53 & GRU & Sao Paulo/Guarulhos & 26.368 .861 \\
\hline 72 & Colombia & BOG & Bogotá/Eldorado & 20.327 .901 \\
\hline 90 & Brasil & CGH & Sao Paulo/Congonhas & 16.687 .731 \\
\hline 91 & Brasil & BSB & Brasilia/International & 15.801 .122 \\
\hline 96 & Brasil & GIG & Río de Janeiro/Galeao & 15.184 .350 \\
\hline
\end{tabular}

Fuente: Airports Council Internacional. 100 Aeropuertos con más pasajeros en el mundo. 
Cuadro 5. Correlaciones población ciudades/pasajeros aeroportuarios. Año 2010.

\begin{tabular}{|l|c|c|}
\hline & $\begin{array}{c}\text { POB. TOTAL/ PASAJEROS } \\
\text { TOTALES. Correlac. simple }\end{array}$ & $\begin{array}{c}\text { RANGO. DEM./RANGO } \\
\text { AEROP. Corr. Spearman }\end{array}$ \\
\hline Brasil & 0,97 & 0,42 \\
\hline México & 0,90 & 0,18 \\
\hline Argentina & 0,99 & $-0,05$ \\
\hline Colombia & 0,98 & 0,54 \\
\hline Perú & 0,99 & $-0,46$ \\
\hline Chile & 0,99 & 0,25 \\
\hline Ecuador & 0,92 & 0,25 \\
\hline $\begin{array}{c}\text { TOTAL AMÉRICA LATINA } \\
\text { (354 ciudades; todos los países) }\end{array}$ & 0,88 & 0,58 \\
\hline
\end{tabular}

Fuente: Elaboración propia a partir del volumen demográfico y el tráfico de pasajeros de los aeropuertos.

Figura 1. Gráfico de dispersión. Relación entre la población total y el tráfico aéreo. Año 2010, millones de habitantes y de pasajeros.

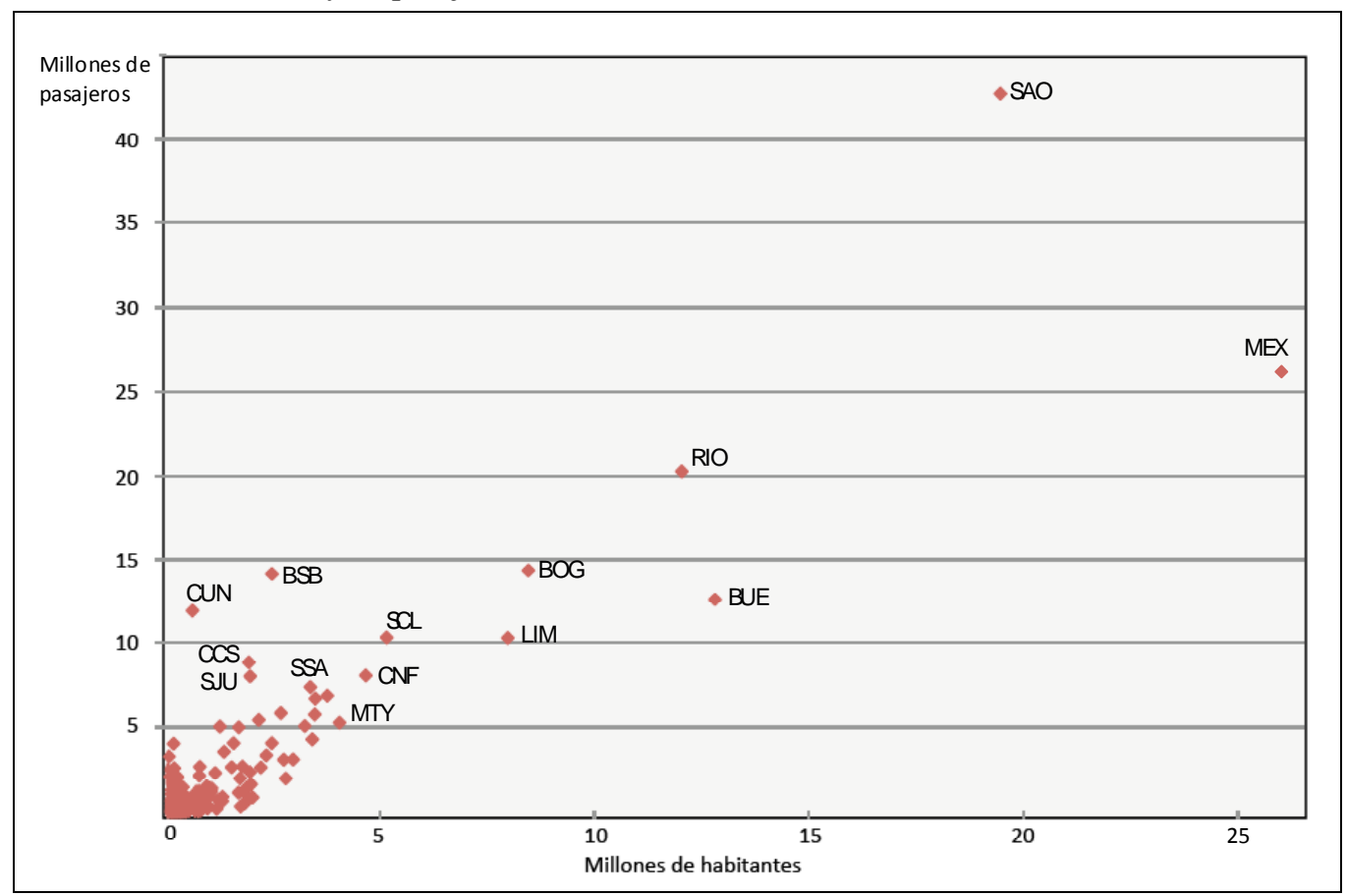

Fuente: Elaboración propia a partir de los resultados de la investigación. 
Figura 2. Gráfico de dispersión. Relación entre el rango demográfico y el aeroportuario (2010).

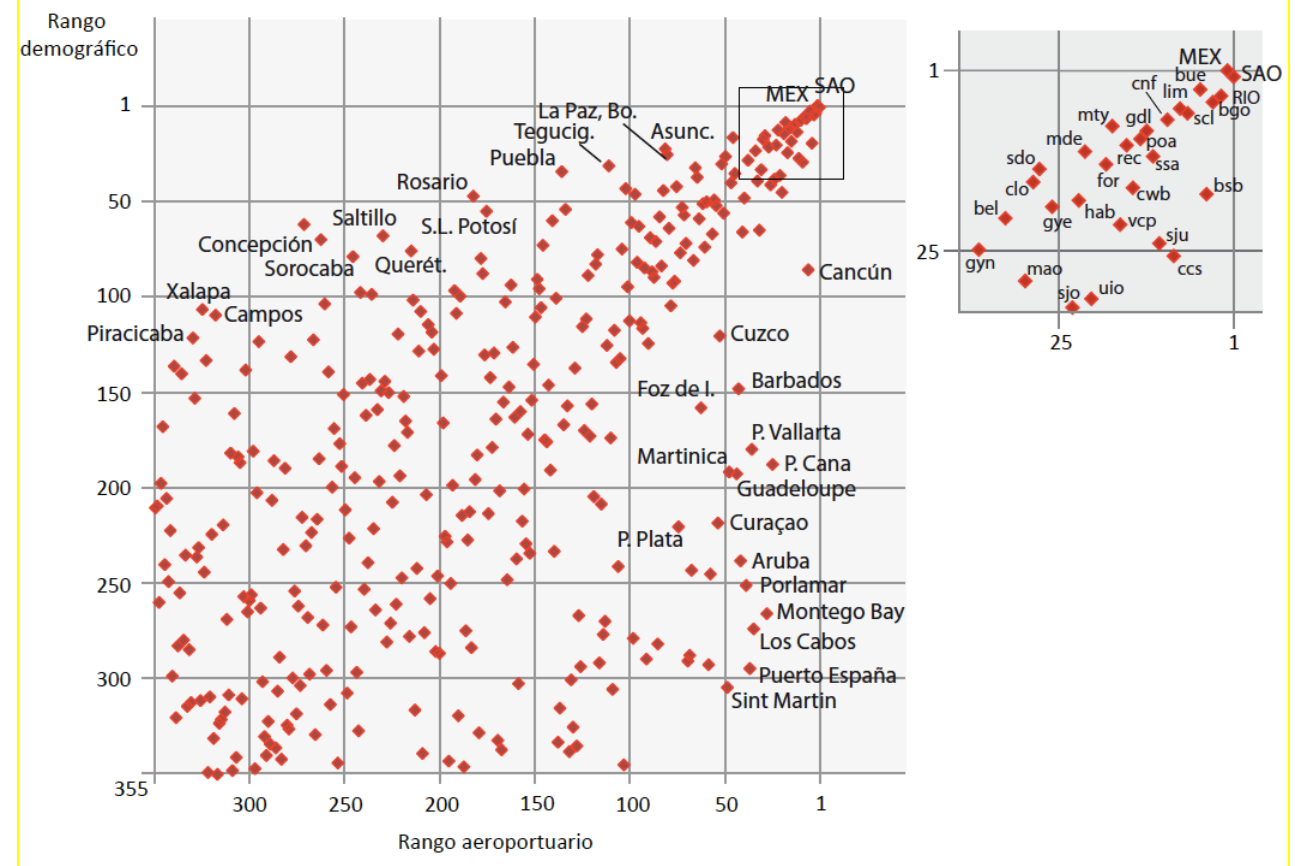

Nota: Rango= lugar que ocupa en una clasificación ordinal de datos.

Fuente: Elaboración propia a partir de los resultados de la investigación.

Para explicar esta falta de correspondencia entre el rango demográfico y el aeroportuario, los estudios de transporte aéreo sugieren al menos dos tópicos: el primero es la existencia de hubs, centros de concentración/dispersión de tráfico aéreo que obedecen, ante todo, a la rentabilidad de la explotación comercial. El segundo tópico son las condiciones de conectividad interna de los sistemas, en particular, la accesibilidad terrestre de los aeropuertos y la accesibilidad generada por las redes domésticas de transporte aéreo. Estos dos argumentos sugieren que no todas las ciudades ni todos aeropuertos tienen capacidad para generar un tráfico cuya explotación sea rentable pero, a nuestro juicio, también son insuficientes para explicar la naturaleza de las relaciones entre los sistemas aeroportuario y urbano latinoamericanos. Aquí sostenemos que el transporte aéreo puede tener un rol territorial específico al dotar a la red de ciudades de los distintos países de una coherencia interna que en algunos casos es imposible obtener con medios de comunicación terrestres, bien porque son insuficientes, bien porque se trata de localizaciones francamente aisladas. Mantenemos, por lo tanto una de las premisas fundamentales de este trabajo en el sentido de que el análisis de la conectividad doméstica del sistema de transporte aéreo puede ser una evidencia de cómo se desarrolla la organización territorial de cada Estado. 
Un estudio anterior sobre las redes domésticas de transporte aéreo (Córdoba y Gago, 2010) ya evidenciaba los siguientes hechos destacados:

1.- Existen países que, por sus dimensiones, carecen de redes domésticas de transporte aéreo: Dominica, Sta. Lucía, Barbados.

2.- Muchos países, generalmente pequeños, tienen redes domésticas de mero desenclave local. Un aeropuerto central, más o menos importante, conecta con núcleos modestos de nula o difícil conectividad superficial: Belice, Guatemala, Salvador, Nicaragua, Panamá, Guyana, Suriname, Guayana Francesa, Paraguay y Uruguay son representativos.

3.- Existen países pequeños, con redes más o menos complejas potenciadas por la insularidad pero, en todo caso, con evidencia de un aeropuerto con funciones centrales dominantes (Curaçao para las Antillas Holandesas; Fort-de-France para las Antillas francesas; San Juan para Puerto Rico, las Islas Vírgenes americanas y Vieques; Providenciales para las Turks y Caicos; Grand Cayman en las Islas Cayman. En algunos casos se trata de relaciones meramente bipolares con un aeropuerto dominante (Trinidad con Tobago, St. Kitts con Nevis y Antigua con Barbuda).

4.- En ciertos casos, la conexión exterior se ha revelado muy fuerte a partir de ciertos centros, generalmente turísticos, mientras que la conectividad doméstica entre ellos es meramente complementaria o inexistente. Es el caso particular de Jamaica, la República Dominicana e incluso Haití y Costa Rica, que ha formado parte del segundo grupo mencionado hasta la internacionalización del aeropuerto de Liberia. Para todo este grupo de países los cambios más relevantes desde 1970 han consistido en la apertura de servicios de desenclave a partir de los aeropuertos principales hacia nuevos destinos generalmente potenciados por el desarrollo turístico.

Las redes domésticas de los países grandes ofrecen, lógicamente, una complejidad mayor y merecen un mayor detenimiento (cuadro 6). Su análisis, sin duda, señala algunos aspectos interesantes, que reflejan cómo el transporte aéreo puede perpetuar los tradicionales desajustes territoriales o, por el contrario, ayudar a una dispersión y reequilibrio en las dinámicas de interacción y movilidad.

En términos generales los datos muestran un aumento de la conectividad media desde 1970, pero con un cierto descenso de la misma entre 1996 y 2011. Este desajuste, sin duda dificulta la cohesión territorial, pero en parte debe interpretarse a la luz de la intensificación en la creación de hubs aeroportuarios y, solo en algunos casos, por la desaparición de determinadas rutas aéreas y por la mejora de las redes de transporte superficial. En términos generales los datos también muestran un sistema aéreo ya consolidado en 1970, que aunque ha sufrido transformaciones durante el periodo analizado, ya anticipaba una alta polarización del tráfico en las capitales nacionales. Puede decirse también que los datos son coherentes con el creciente desarrollo del sector aéreo, lo que se aprecia en la intensificación importante de las frecuencias medias, especialmente en los casos de Brasil, Colombia, México y Perú donde se sobrepasan los 350 vuelos mensuales por conexión (principales ciudades de cada sistema doméstico). 
El análisis pormenorizado, país por país, revela además aspectos claves para valorar el proceso. En primer lugar, Argentina y Chile manifiestan la importante centralidad de sus capitales evidenciando, también, la peculiar configuración meridiana de ambos territorios nacionales; las capitales de estos dos países distan mucho de sus seguidoras, Córdoba y Mendoza $\left(\mathbf{C K}^{9}=12\right)$ y Antofagfasta e Iquique $(\mathbf{C K}=45,5)$. En el caso chileno, la organización actual del transporte aéreo es un calco de la de 1970, presentando unas conectividades medias muy estables, con coeficientes de conectividad muy aceptables tanto en la muestra de las principales ciudades, como en los cálculos que incluyen un mayor número $(n=12)$. Un análisis pormenorizado de las matrices de conectividad muestra dos ejes aéreos que dividen en territorio en dos mitades y que tienen a Santiago como elemento central. Argentina, por su parte, es el país de todos los analizados con coeficientes de conectividad más bajos, observándose un descenso de la interconexión media entre 1996 y 2011, lo que, sin duda, no ayuda a la vertebración territorial de un país tan extenso y con unas redes de carreteras insuficientes (sirva de ejemplo los escasos $800 \mathrm{Km}$. de vías rápidas que alberga el territorio). La cohesión territorial del Estado argentino, sin duda, se ve poco facilitada por unos coeficientes de conexión que sólo en tres de sus cinco ciudades principales alcanzan el nivel 50 (Buenos Aires, Córdoba y Mendoza; $\mathrm{n}=5$ ).

También en Venezuela y Perú perviven redes domésticas polarizadas por sus capitales. Caracas $(\mathbf{C K}=100)$ y Lima $(\mathbf{C K}=92,9$ para un total de 15 ciudades) tienen coeficientes de conexión notablemente superiores a los de las ciudades que les siguen en el ranking de conectividad doméstica (Valencia y Maracaibo CK=50 y Cuzco $\mathbf{C K}=28,6$ ). Los sistemas urbanos de estos países requerirían de una red aérea más tupida, incluso manteniendo la centralidad de las capitales, habida cuenta de las escasas redes terrestres disponibles (Perú: $10 \mathrm{~km} / \mathrm{km}^{2}$, Colombia: $11 \mathrm{~km} / \mathrm{km}^{2}$ ) y de la existencia de amplios territorios todavía escasamente antropizados o muy inaccesibles, para cuyo acceso se hace indispensable el avión.

Ecuador y Bolivia han mantenido redes que se articulan entre grupos de ciudades dominantes, aunque con matices. Los datos indican que la red aérea de Ecuador estaba muy poco consolidada en 1970, aunque mostrando ya, en aquel momento, el protagonismo bicefálico de Quito y Guayaquil.

\footnotetext{
${ }^{9}$ El coeficiente de conectividad (CK) es un cálculo simple que relaciona la conectividad real de un nodo (las relaciones reales de ese nodo) con la conectividad potencial (el número de nodos susceptibles de estar conectados con él, esto es, todos los nodos del sistema menos él mismo y aquéllos que están demasiado cerca como para que se establezca una relación aérea con ellos). Puede entenderse como un simple porcentaje de forma que 100 quiere decir que hay conexión total y 50 que existe la mitad de las conexiones posibles. Algunos trabajos realizados utilizando este tipo de indicador son: Gago, 2003; Córdoba y Gago, 2002 y 2010.
} 
Por su parte, en Bolivia se descubre un sistema bastante consolidado en 1970, pivotando sobre tres ciudades, Cochabamba, Santa Cruz y La Paz (CK=66,7 en los tres casos), ampliándose el grupo a las ciudades de Sucre y Tarija. Sin embargo, núcleos muy significativos de la riqueza en recursos de Bolivia como Potosí y Oruro han perdido toda la conectividad aérea, incidiéndose por lo tanto en su aislamiento, aún con las salvedades que puedan hacerse, como que se encuentran relativamente poco distantes de las ciudades de Cochabamba o Sucre ${ }^{10}$.

Tanto en México como en Colombia, las redes domésticas siguen muy polarizadas por la centralidad geográfica de sus capitales, Ciudad de México (CK=89,7 para un total de 40 ciudades) y Bogotá ( $\mathbf{C K}=86,9$ para 24 ciudades). En ambos países, se observa además que la conectividad general de las principales ciudades del sistema urbano es muy alta. En México esta conectividad se ha incrementado hasta el 81,1, revelándose además el basculamiento hacia el norte de la movilidad, tanto por el dinamismo de Monterrey como por la importante conectividad de Tijuana y Chihuahua. Además, observamos otros centros de gravedad regional en el interior del país como Guadalajara (con CK elevados), y León y en el Sur del país, Mérida. En el caso de México, todos estos aspectos nos hablan, sin duda, de un proceso de interrelación territorial creciente, donde la red de carreteras ha conocido, también un proceso de modernización asombroso. En la actualidad este país ocupa el puesto 17 del mundo por número de carreteras pavimentadas, disponiendo de más de $6.200 \mathrm{~km}$ de autopistas o autovías ${ }^{11}$. En cualquier caso, parece que el eje territorial en México se ha desplazado hacia el Norte en donde sus ciudades han alcanzado un protagonismo creciente que también se observa en lo económico y, como veremos en el epígrafe siguiente, en su proyección internacional.

En cuanto a Colombia se aprecia una conectividad media-alta entre las principales ciudades del sistema en donde destaca, además de Bogotá, Medellín. Sin embargo la conectividad de esta última, junto con otras como Cali y Cartagena, disminuye considerablemente si analizamos la conectividad conjunta de las 24 ciudades seleccionadas.

Brasil, por último, merece una atención diferenciada debido a la gran complejidad de su red doméstica de transporte aéreo y a la originalidad de su sistema urbano: tremenda extensión del territorio y elevado número de núcleos que participan en su red de conexiones aéreas, en la que además los enlaces regionales y locales de desenclave son muy importantes. Estos últimos presentan coeficientes de conectividad altos entre las ciudades principales $(\mathbf{C K}=83,8, \mathrm{n}=15)$ y medio-altos para una muestra de 33 ciudades, donde además de las ciudades de mayor volumen demográfico se incluyen también las capitales de los Estados.

\footnotetext{
${ }^{10}$ Distancias medias: Oruro- Cochabamba $=216 \mathrm{~km}$ (3.30 horas); Potosí- Sucre $=155 \mathrm{~km}$ (2.30 horas).

${ }^{11}$ Fuente: CIA Factbook, 2013.
} 
Cuadro 6. Coeficientes de conexión aérea doméstica (CK) de América Latina (países y ciudades seleccionadas).

\begin{tabular}{|l|r|r|r|r|r|}
\hline ARGENTINA & \multicolumn{3}{|c|}{ CK. N= 5 } & \multicolumn{2}{|c|}{ C.K. N= 26 } \\
\hline \multicolumn{1}{|c|}{ AÑo } & $\mathbf{1 9 7 0}$ & $\mathbf{1 9 9 6}$ & $\mathbf{2 0 1 1}$ & $\mathbf{1 9 9 6}$ & $\mathbf{2 0 1 1}$ \\
\hline ARG. TOTAL & $\mathbf{7 0 , 0}$ & $\mathbf{9 0 , 0}$ & $\mathbf{5 0 , 0}$ & $\mathbf{2 1 , 8}$ & $\mathbf{1 0 , 6}$ \\
\hline Buenos Aires & 100,0 & 100,0 & 100,0 & 96,0 & 96,0 \\
\hline Córdoba & 100,0 & 100,0 & 50,0 & $\mathbf{4 8 , 0}$ & 12,0 \\
\hline Mendoza & 50,0 & 100,0 & 50,0 & 40,0 & 12,0 \\
\hline Rosario & 50,0 & 75,0 & 25,0 & 12,0 & 4,0 \\
\hline Tucumán & 50,0 & 75,0 & $\mathbf{2 5 , 0}$ & 16,0 & 4,0 \\
\hline Vuelos/conexión & $\mathbf{5 6 , 6}$ & 185,5 & 323,7 & 66,9 & $\mathbf{7 4 , 0 4}$ \\
\hline
\end{tabular}

\begin{tabular}{|l|r|r|r|r|r|}
\hline COLOMBIA & \multicolumn{3}{|c|}{ CK. N= 5 } & \multicolumn{2}{|c|}{ C.K. N= 24 } \\
\hline \multicolumn{1}{|c|}{ AÑo } & $\mathbf{1 9 7 0}$ & $\mathbf{1 9 9 6}$ & $\mathbf{2 0 1 1}$ & $\mathbf{1 9 9 6}$ & $\mathbf{2 0 1 1}$ \\
\hline COL. TOTAL & $\mathbf{8 0 , 0}$ & $\mathbf{1 0 0 , 0}$ & $\mathbf{7 0 , 0}$ & $\mathbf{1 9 , 5}$ & $\mathbf{1 2 , 3}$ \\
\hline Barranquilla & 100,0 & 100,0 & 50,0 & 30,4 & 8,70 \\
\hline Bogota & 75,0 & 100,0 & 100,0 & $\mathbf{8 2 , 6}$ & 86,96 \\
\hline Cali & 75,0 & 100,0 & 50,0 & 39,1 & 13,04 \\
\hline Cartagena & 100,0 & 100,0 & 50,0 & 26,1 & 13,04 \\
\hline Medellin & 80,0 & 100,0 & 100,0 & 69,6 & 47,83 \\
\hline Vuelos/conexión & 123,2 & 217,8 & 516,9 & 129,4 & 236,7 \\
\hline
\end{tabular}

\begin{tabular}{|l|r|r|r|}
\hline \multicolumn{1}{|c|}{ BOLIVIA } & \multicolumn{3}{|c|}{ CK. N=7 } \\
\hline \multicolumn{1}{|r|}{$\mathbf{1 9 7 0}$} & $\mathbf{1 9 9 6}$ & $\mathbf{2 0 1 1}$ \\
\hline BOL. TOTAL & $\mathbf{5 5 , 5}$ & $\mathbf{6 6 , 6}$ & $\mathbf{5 2 , 8}$ \\
\hline Cochabamba & 100,0 & 66,7 & 66,7 \\
\hline La Paz & 33,3 & 100,0 & 66,7 \\
\hline Oruro & 16,7 & 0,0 & 0,0 \\
\hline Potosi & 16,7 & 16,7 & 0,0 \\
\hline Santa Cruz & 66,7 & 83,3 & 66,7 \\
\hline Sucre & 50,0 & 66,7 & 50,0 \\
\hline Tarija & $\mathbf{5 0 , 0}$ & 66,7 & 50,0 \\
\hline Vuelos/conexión & 20,4 & 70,59 & 90 \\
\hline
\end{tabular}

\begin{tabular}{|c|c|c|c|c|c|}
\hline \multirow{2}{*}{\begin{tabular}{|l|} 
BRASIL \\
AÑo
\end{tabular}} & \multicolumn{3}{|c|}{ CK. $N=15$} & \multicolumn{2}{|c|}{ C.K. $\mathrm{N}=33$} \\
\hline & 1970 & 1996 & 2011 & 1996 & 2011 \\
\hline BRASIL TOTAL & 57,1 & 71,9 & 83,8 & 40,3 & 39,3 \\
\hline Belem & 57,1 & 78,6 & 64,3 & 56,3 & 40,6 \\
\hline Belo Horizonte & 42,9 & 50,0 & 100,0 & 53,1 & 78,1 \\
\hline Brasilla & 71,4 & 92,9 & 100,0 & 78,1 & 87,5 \\
\hline Cuiaba & 28,6 & 42,9 & 64,3 & 50,0 & 40,6 \\
\hline Curitiba & 28,6 & 71,4 & 100,0 & 59,4 & 68,8 \\
\hline Florianopolls & 21,4 & 57,1 & 50,0 & 46,9 & 25,0 \\
\hline Fortaleza & 85,7 & 78,6 & 85,7 & 56,3 & 53,1 \\
\hline Manaus & 50,0 & 71,4 & 85,7 & 46,9 & 53,1 \\
\hline Natal & 42,9 & 64,3 & 57,1 & 40,6 & 28,1 \\
\hline Porto Alegre & 50,0 & 57,1 & 85,7 & 56,3 & 59,4 \\
\hline Recife & 78,6 & 78,6 & 92,9 & 65,6 & 59,4 \\
\hline Rio de Janeiro & 100,0 & 92,9 & 100,0 & 84,4 & 93,8 \\
\hline Salvador & 57,1 & 92,9 & 92,9 & 59,4 & 62,5 \\
\hline Sao Paulo & 100,0 & 100,0 & 100,0 & 100,0 & 96,9 \\
\hline Vitoria (BR) & 42,9 & 50,0 & 78,6 & 34,4 & 43,8 \\
\hline Vuelos/conexión & 71 & 166,6 & 378 & 102,3 & 220,87 \\
\hline
\end{tabular}

\begin{tabular}{|l|r|r|r|}
\hline \multicolumn{1}{|c|}{ ECUNO } & \multicolumn{3}{|c|}{ CK. N=8 } \\
\hline AÑ & $\mathbf{1 9 7 0}$ & $\mathbf{1 9 9 6}$ & $\mathbf{2 0 1 1}$ \\
\hline ECUAD. TOTAL & $\mathbf{3 , 6}$ & $\mathbf{7 , 4}$ & $\mathbf{3 5 , 7}$ \\
\hline Cuenca & 0,0 & 12,5 & 28,6 \\
\hline Esmeraldas & 0,0 & 0,0 & 14,3 \\
\hline Guayaquil & 14,3 & 12,5 & 57,1 \\
\hline Loja & 0,0 & 0,0 & 28,6 \\
\hline Manta & 0,0 & 0,0 & 14,3 \\
\hline Portoviejo & 0,0 & 0,0 & 14,3 \\
\hline Quito & 14,3 & 25,0 & 100,0 \\
\hline Santa Rosa (EC) & 0,0 & 0,0 & 28,6 \\
\hline Vuelos/conexión & 82,0 & 188,75 & 166,55 \\
\hline
\end{tabular}

\begin{tabular}{|l|r|r|r|r|r|}
\hline CHILE & \multicolumn{3}{|c|}{ CK. N=7 } & \multicolumn{2}{c|}{ C.K. N= 12 } \\
\hline \multicolumn{1}{|c|}{ ANNO } & $\mathbf{1 9 7 0}$ & $\mathbf{1 9 9 6}$ & $\mathbf{2 0 1 1}$ & $\mathbf{1 9 9 6}$ & $\mathbf{2 0 1 1}$ \\
\hline CHILE TOTAL & $\mathbf{5 2 , 3}$ & $\mathbf{5 7 , 1}$ & $\mathbf{5 7 , 1}$ & $\mathbf{3 7 , 1}$ & $\mathbf{3 8 , 6}$ \\
\hline Antofagasta & $\mathbf{5 0 , 0}$ & $\mathbf{5 0 , 0}$ & $\mathbf{5 0 , 0}$ & $45, \mathbf{5}$ & $\mathbf{4 5 , 5}$ \\
\hline Arica & 50,0 & 50,0 & 50,0 & 27,3 & 36,4 \\
\hline Concepción & 50,0 & 50,0 & 50,0 & 45,5 & 27,3 \\
\hline Iquique & 50,0 & 50,0 & 50,0 & 36,4 & 45,5 \\
\hline Puerto Montt & 33,3 & 50,0 & 50,0 & 36,4 & 36,4 \\
\hline Santiaqo & 100,0 & 100,0 & 100,0 & 100,0 & 100,0 \\
\hline Temuco & 33,3 & 50,0 & 50,0 & 54,5 & 36,4 \\
\hline Vuelos/conexión & 41 & 139,27 & 158,6 & $\mathbf{9 5 , 1 7}$ & 110,5 \\
\hline
\end{tabular}

\begin{tabular}{|l|r|r|r|r|r|}
\hline MÉXICO & \multicolumn{3}{|c|}{ CK. N= 8 } & \multicolumn{2}{c|}{ C.K. N= 40 } \\
\hline \multicolumn{1}{|c|}{ AÑO } & $\mathbf{1 9 7 0}$ & $\mathbf{1 9 9 6}$ & $\mathbf{2 0 1 1}$ & $\mathbf{1 9 9 6}$ & $\mathbf{2 0 1 1}$ \\
\hline MEX. TOTAL & $\mathbf{6 7 , 8}$ & $\mathbf{7 6 , 7}$ & $\mathbf{8 1 , 1}$ & $\mathbf{2 0 , 2}$ & $\mathbf{1 3 , 2}$ \\
\hline Cdad. Juárez & 71,4 & 71,4 & 85,7 & $\mathbf{2 5 , 6}$ & 17,9 \\
\hline Cdad. México & 100,0 & 100,0 & 100,0 & $\mathbf{9 2 , 3}$ & 89,7 \\
\hline Chihuahua & 71,4 & 85,7 & 71,4 & 28,2 & 20,5 \\
\hline Guadalajara & 57,1 & 100,0 & 85,7 & 59,0 & 38,4 \\
\hline León/Bajio & 28,6 & 71,4 & 42,8 & 20,5 & $\mathbf{7 , 7}$ \\
\hline Mérida & 42,9 & 14,3 & 42,8 & 17,9 & 15,3 \\
\hline Monterrey & 100,0 & 85,7 & 100,0 & 51,2 & 51,2 \\
\hline Tijuana & 71,4 & 85,7 & 85,7 & 53,9 & 46,1 \\
\hline Vuelos/conexión & 46,1 & 242,1 & 490,15 & 115,0 & $132, \mathbf{2 2}$ \\
\hline
\end{tabular}

\begin{tabular}{|l|r|r|r|r|r|}
\hline PERU & \multicolumn{3}{|c|}{ CK. N=6 } & \multicolumn{2}{c|}{ C.K. N= 15 } \\
\hline \multicolumn{1}{|c|}{ AN̂́O } & $\mathbf{1 9 7 0}$ & $\mathbf{1 9 9 6}$ & $\mathbf{2 0 1 1}$ & $\mathbf{1 9 9 6}$ & $\mathbf{2 0 1 1}$ \\
\hline PERÚ TOTAL & $\mathbf{4 6 , 6}$ & $\mathbf{5 6 , 6}$ & $\mathbf{5 0 , 0}$ & $\mathbf{2 3 , 8}$ & $\mathbf{1 7 , 6}$ \\
\hline Arequipa & 20,0 & 40,0 & 40,0 & $\mathbf{2 8 , 6}$ & $\mathbf{2 1 , 4}$ \\
\hline Cuzco & 20,0 & 60,0 & 60,0 & $\mathbf{2 8 , 6}$ & $\mathbf{2 8 , 6}$ \\
\hline Iquitos & 40,0 & 40,0 & 40,0 & 28,6 & 21,4 \\
\hline Lima & 100,0 & 100,0 & 100,0 & 85,7 & 92,9 \\
\hline Plura & 40,0 & 40,0 & 20,0 & 21,4 & 7,1 \\
\hline Trujillo & 60,0 & 60,0 & 20,0 & 35,7 & 14,2 \\
\hline Vuelos/conexión & 47,2 & 117 & 351 & 91,4 & 177,7 \\
\hline
\end{tabular}

\begin{tabular}{|l|r|r|r|}
\hline VENEZUELA & \multicolumn{3}{|c|}{ CK. N=7 } \\
\hline \multicolumn{1}{|r|}{ AÑO } & $\mathbf{1 9 7 0}$ & $\mathbf{1 9 9 6}$ & $\mathbf{2 0 1 1}$ \\
\hline VENEZ. TOTAL & $\mathbf{4 2 , 9}$ & $\mathbf{5 2 , 3}$ & $\mathbf{5 4 , 7}$ \\
\hline Barcelona & 50,0 & 33,3 & 33,3 \\
\hline Barquisimeto & 33,3 & 33,3 & 33,3 \\
\hline Caracas & 83,3 & 100,0 & 100,0 \\
\hline Maracaibo & 33,3 & 50,0 & $\mathbf{5 0 , 0}$ \\
\hline Maturín & 50,0 & 33,3 & 33,3 \\
\hline Cdad. Guayana & 50,0 & 66,7 & 66,7 \\
\hline Valencia & 0,0 & 50,0 & 50,0 \\
\hline Vuelos/conexión & 73,2 & 91,27 & 147,9 \\
\hline
\end{tabular}

Fuente: Elaboración propia a partir de Guías ABC World Airways, 1970 y bases de Datos, COMBER International, sobre frecuencias mundiales de transporte aéreo, 1996 y 2011. 
Sao Paulo es cabeza primada de la jerarquía aeroportuaria manteniendo un CK elevado desde 1970. Le siguen en importancia Río de Janerio y especialmente Brasilia y Belo Horizonte, que configuran un cuarteto director que define un modelo de red compleja y muy tupida más parecido al de Estados Unidos que al de cualquier otro país latinoamericano. Desde 1970, además, la conectividad aérea del sistema doméstico principal ha aumentado de 57,1 a $83,8 \%$ dando una fuerte cohesión al sistema urbano de equilibrio regional. En este sentido, la mayor parte de las grandes ciudades brasileñas (Porto Alegre, Recife, Salvador, Fortaleza, Curitiba, Belem y Manaos) deben considerarse emergentes de cara a un hipotético mercado global y cabe añadir a esta condición otras ciudades no tan grandes pero que se definen como centros regionales de elevada conectividad doméstica (Florianópolis y Vitoria entre ellas, pero sobre todo Cuiabá y Natal). Brasil presenta, además una intensidad de conexión (vuelos/frecuencia), elevada, igualando casi a Colombia, pero con una conectividad mucho más amplia que sin duda favorece la cohesión territorial y la competitividad en los mercados globales.

Las evidencias empíricas presentadas hasta el momento, referidas a las características y evolución de las redes domésticas de transporte aéreo latinoamericano permiten, en definitiva, sugerir varias trayectorias, en algunos casos, contrapuestas:

1.- La persistencia de fuertes centralidades en ciertos nodos privilegiados que, asociados a condiciones de macrocefalia en el sistema urbano, perpetúan sistemas manifiestamente centralistas que limitan la emergencia de centros regionales en el escenario nacional. Es, ante todo, el caso de Perú y Argentina, pero también el caso más velado de Venezuela, Colombia, Chile e, incluso, México.

2.- Una tendencia a la participación cada vez más activa de los centros regionales en la estructuración del territorio nacional. Es el caso de Brasil y un fenómeno que empieza a vislumbrarse en México, país que se encontraría actualmente entre los dos modelos debido, sobre todo, a las mejoras de su red viaria y a la consolidación en el centro del país del protagonismo de Guadalajara y León, a la vez que han eclosionado en el Norte importantes centros regionales como Monterrey, Tijuana y Chihuahua.

\section{TRANSPORTE AÉREO INTERNACIONAL Y SISTEMA URBANO}

En este apartado se profundiza en la conectividad aérea internacional de las principales ciudades latinoamericanas como un elemento para valorar su inserción en el sistema mundial de ciudades. En este sentido, la valoración del nivel de conectividad internacional y la direccionalidad de conexiones y frecuencias, nos permite ver la dimensión y el alcance que tiene el transporte aéreo en la región como elemento globalizador.

Un mapa sencillo de accesibilidad muestra que la inmensa mayoría de las ciudades latinoamericanas con más de 50.000 habitantes se encuentran en un radio de acción de un aeropuerto internacional inferior a $250 \mathrm{Km}$. Sin embargo, demasiadas ciudades se sitúan fuera de un radio de acción inferior a $100 \mathrm{Km}$. (figura 3). Siendo muy defi- 
cientes las condiciones de conectividad superficial en la mayoría de los países latinoamericanos, estos umbrales sugieren que la conectividad internacional de la mayoría de las ciudades es modesta y depende de forma prácticamente exclusiva de las redes domésticas de transporte aéreo, tal como sostenemos en el capítulo anterior. Ya ha sido señalado anteriormente la escasa relevancia de los aeropuertos latinoamericanos si los comparamos con los más importantes del mundo; a continuación se valorará la direccionalidad de las conexiones entre pares de ciudades y frecuencias como signo de la inclusión de los sistemas microrregionales en el contexto global (cuadro 7).

Un primer análisis permite destacar la notable concentración de los flujos en dos direcciones, hacia el interior de la región en la que se ubican los nodos y hacia Norteamérica; en este sentido, la direccionalidad indica una clara polarización de componente Norte-Sur, concentrando Norteamérica más conexiones y frecuencias (38,8\% de las conexiones; $33,8 \%$ de los vuelos o frecuencias), que cada una de las regiones identificadas por sí mismas. El peso que adquiere la región Caribe también es considerable, aunque debe ser considerado en su medida, en virtud del copioso número de pequeñas islas con salida internacional, gran parte de ellas dentro de la propia región a la que pertenecen; parece también reseñable que la conexión de las regiones contiguas en el interior de América Latina es más bien reducida, lo que denota la manifiesta debilidad de los flujos interregionales. También puede avanzarse que un análisis más detallado de la direccionalidad, que por motivos de espacio este artículo no permite, indicaría que la conectividad internacional está marcada, además de por la vecindad intrarregional, por una impronta cultural y colonial muy importante, con relaciones entre los territorios vinculados a una misma exmetrópoli, existiendo en cambio una conectividad escasa de los territorios de colonización no hispana con Centroamérica y Mesoamérica, excepto las conexiones de las Antillas Holandesas con Venezuela y Colombia.

Igual que las islas Caribeñas, México y los países centroamericanos muestran una importante tendencia en la concentración de sus flujos aéreos hacia Norteamérica (60,3\% de las conexiones de la región), por encima de los que se destinan a su propia región (17,5\%). En los países de América del Sur, tanto los del Norte como los del Cono Sur, la polarización norteamericana se relaja siendo las conexiones intrarregionales más importantes. Cabe destacar, también, cómo los lazos con la región europea adquieren aquí cierto protagonismo, mucho más si se consideran las conexiones, que la totalidad de los vuelos. En este sentido parece interesante resaltar la importancia que tiene para la proyección de los territorios en los circuitos globales traspasar los límites regionales; en este caso, el alcance extracontinental es muy limitado, reduciéndose en términos generales solo a Europa, continente con el que existen lazos históricos y culturales muy importantes. Son mucho más escasas las conexiones con otros continentes, casi testimoniales, adquiriendo por ello un valor altamente estratégico, como es el caso de las relaciones con el Sur de África (Rep. Sudafricana y Angola), y hacia Oriente Medio y hacia Asia (ruta Tijuana-Tokio). 
Figura 3. Sistema urbano y accesibilidad a un aeropuerto internacional.

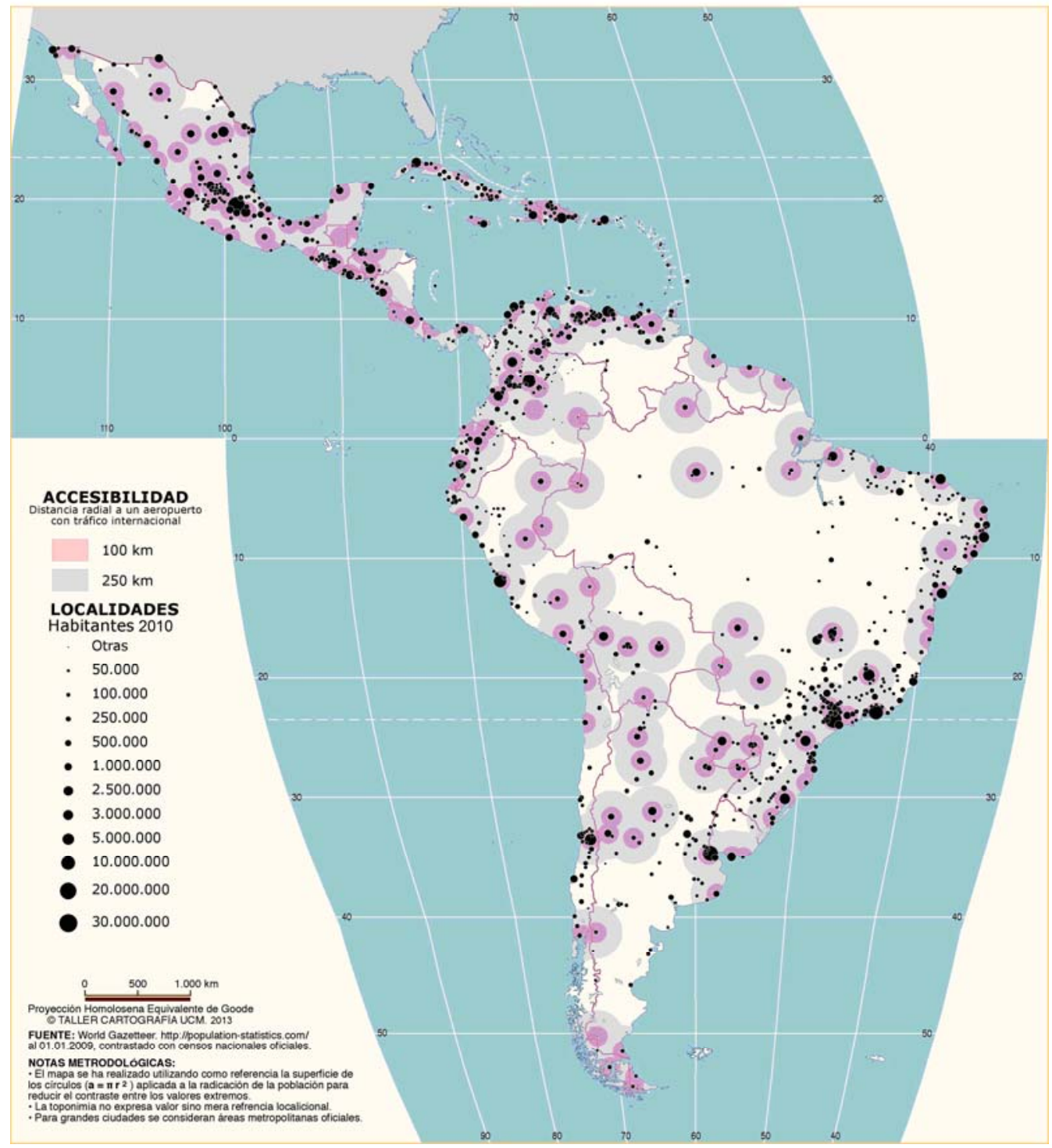

Fuente: Elaboración propia. 
Cuadro 7. Direccionalidad de conexiones y frecuencias internacionales. Latinoamérica 2011.

\begin{tabular}{|c|c|c|c|c|c|c|c|c|}
\hline & Caribe & $\begin{array}{c}\text { Mex.+ Am. } \\
\text { Central }\end{array}$ & $\begin{array}{c}\text { Cono Sur } \\
+ \text { Brasil }\end{array}$ & Mesoamérica & Norteam. & Europa & RESTO & TOTAL \\
\hline Canibe & 17,3 & 1,4 & 0,4 & 2,0 & 16,3 & 5,4 & 0,15 & 589 \\
\hline Mex.+ Centroam. & 1,2 & 5,0 & 0,8 & 2,0 & 17,1 & 2,0 & 0,22 & 388 \\
\hline Cono Sur+ Brasil & 0,5 & 0,8 & 4,4 & 2,4 & 2,5 & 3,0 & 1,02 & 200 \\
\hline Mesoamérica & 2,0 & 2,0 & 2,3 & 2,9 & 2,9 & 1,5 & 0,15 & 189 \\
\hline TOTAL AM. LAT. & 21,16 & 9,15 & 7,98 & 9,37 & 38,80 & 12,01 & 1,54 & 1366 \\
\hline \multicolumn{9}{|c|}{ FRECUENCIAS. \% RESPECTO AL TOTAL. TOTAL $=68817$} \\
\hline Caribe & 25,8 & 1,1 & 0,0 & 1,4 & 13,5 & 1,7 & 0,00 & 29942 \\
\hline Mex.+ Centroam. & 1,1 & 6,5 & 0,7 & 2,3 & 15,8 & 0,8 & 0,08 & 18761 \\
\hline Cono Sur+ Brasil & 0,0 & 0,7 & 9,2 & 2,0 & 2,0 & 2,1 & 0,38 & 11310 \\
\hline Mesoamérica & 1,4 & 2,3 & 2,1 & 3,5 & 2,5 & 1,0 & 0,01 & 8804 \\
\hline TOTAL AM. LAT. & 28,29 & 10,70 & 11,99 & 9,18 & 33,80 & 5,55 & 0,48 & \\
\hline \multicolumn{9}{|c|}{ CONEXIONES. \% RESPECTO AL TOTAL DE LA REGIÓN. } \\
\hline Caribe & 40,2 & 3,2 & 1,0 & 4,8 & 37,9 & 12,6 & 0,34 & \\
\hline Mex.+ Centroam. & 4,4 & 17,5 & 2,8 & 7,0 & 60,3 & 7,2 & 0,77 & \\
\hline Cono Sur+ Brasil & 3,5 & 5,5 & 30,0 & 16,5 & 17,0 & 20,5 & 7,00 & \\
\hline Mesoamérica & 14.81 & 14.29 & 16,93 & 21,16 & 20.63 & 11.11 & 1,06 & \\
\hline \multicolumn{9}{|c|}{ CIUDADES SELECCIONADAS: CONEXIONES. \% RESPECTO AL TOTAL. } \\
\hline Buenos Aires & 8,8 & 5,9 & 23,5 & 14,7 & 17,6 & 17,6 & 11,76 & 34 \\
\hline Ciudad de Mex. & 2,6 & 12,8 & 7,7 & 5,1 & 56,4 & 15,4 & & 39 \\
\hline Rio de Janeiro & & 5,3 & 21,1 & 5,3 & 31,6 & 31,6 & 5,26 & 19 \\
\hline Sao Paulo & 2,4 & 4,9 & 12,2 & 9,8 & 26,8 & 31,7 & 12,20 & 41 \\
\hline Bogotáa & 13,8 & 17,2 & 10,3 & 17,2 & 27,6 & 13,8 & & 29 \\
\hline Brasilia & 20,0 & & & 20,0 & 40,0 & 20,0 & & 5 \\
\hline Caracas & 21,9 & 6,3 & 9,4 & 15,6 & 18,8 & 25,0 & 3,13 & 32 \\
\hline Habana & 28,6 & 17,9 & 7,1 & 10,7 & 7,1 & 25,0 & 3,57 & 28 \\
\hline La Paz (BO) & & & 50,0 & 50,0 & & & & 4 \\
\hline Lima & 8,1 & 13,5 & 32,4 & 18,9 & 21,6 & 5,4 & & 37 \\
\hline Cdad. de Panamá & 20,8 & 15,1 & 15,1 & 28,3 & 17,0 & 3,8 & & 53 \\
\hline Quito & 10,0 & 10,0 & & 50,0 & 30,0 & & & 10 \\
\hline San Jose & 6,9 & 31,0 & & 17,2 & 41,4 & 3,4 & & 29 \\
\hline Santiago $(\mathrm{CL})$ & 8,7 & 8,7 & 30,4 & 17,4 & 21,7 & 8,7 & 4,35 & 23 \\
\hline Aruba & 14,3 & 3,6 & 3,6 & 21,4 & 42,9 & 14,3 & & 28 \\
\hline Fort de France & 66,7 & & & 11,1 & 11,1 & 11,1 & & 9 \\
\hline Grand Cayman Is. & 26,7 & 6,7 & & & 66,7 & & & 15 \\
\hline San Juan (PR) & 47,4 & 2,6 & & 2,6 & 44,7 & 2,6 & & 38 \\
\hline Cali & & 20,0 & & 40,0 & 20,0 & 20,0 & & 5 \\
\hline Córdoba & & 14,3 & 57,1 & 14,3 & & 14,3 & & 7 \\
\hline Guadalajara & & 7,1 & & & 92,9 & & & 14 \\
\hline Guayaquil & & 23,1 & 15,4 & 30,8 & 23,1 & 7,7 & & 13 \\
\hline Medellin & 11,1 & 22,2 & & 22,2 & 33,3 & 11,1 & & 9 \\
\hline Monterrey & & & & & 100,0 & & & 7 \\
\hline Porto Aleqre & & & 80,0 & 20,0 & & & & 5 \\
\hline Recife & & & & & 25,0 & 75,0 & & 4 \\
\hline Cancún & 1,7 & 6,7 & & 3,3 & 60,0 & 28,3 & & 60 \\
\hline Montego Bay & 4,0 & & & & 64,0 & 32,0 & & 25 \\
\hline Punta Cana & 12,8 & 2,6 & 5,1 & 5,1 & 35,9 & 38,5 & & 39 \\
\hline San Jose Cabo & & & & & 100,0 & & & 16 \\
\hline
\end{tabular}

CIUDADES SELECCIONADAS:FRECUENCIAS, \% RESPECTO AL TOTAL.

\begin{tabular}{|c|c|c|c|c|c|c|c|c|}
\hline Buenos Aires & 0,4 & 4,0 & 65,8 & 10,6 & 8,8 & 8,8 & 1,53 & 3147 \\
\hline Ciudad de Mex. & 2,0 & 9,0 & 4,3 & 7,6 & 69,0 & 8,2 & & 3152 \\
\hline Rio de Janeiro & & 3,6 & 39,5 & 3,2 & 24,6 & 27,5 & 1,64 & 856 \\
\hline Sao Paulo & 0,1 & 4,7 & 38,5 & 11,2 & 19,8 & 21,0 & 4,72 & 2837 \\
\hline Bogotá & 4,1 & 21,9 & 10,8 & 33,4 & 23,2 & 6,5 & & 2131 \\
\hline Brasilia & 3,2 & & & 28,2 & 47,6 & 21,0 & & 124 \\
\hline Caracas & 20,6 & 12,1 & 7,7 & 22,2 & 22,2 & 15,1 & 0,16 & 1282 \\
\hline Habana & 8,3 & 49,3 & 2,1 & 11,7 & 5,2 & 23,2 & 0,32 & 617 \\
\hline La Paz (BO) & & & 44,1 & 55,9 & & & & 111 \\
\hline Lima & 2,1 & 15,4 & 36,8 & 23,3 & 16,5 & 5,8 & & 2125 \\
\hline Cdad. de Panamá & 16,9 & 23,0 & 11,2 & 32,5 & 15,1 & 1,3 & & 3263 \\
\hline Quito & 3,5 & 9,4 & & 65,0 & 22,2 & & & 663 \\
\hline San Jose & 3,2 & 50,7 & & 12,3 & 31,8 & 2,0 & & 1553 \\
\hline Santiago (CL) & 0,5 & 6,2 & 55,6 & 20,0 & 10,9 & 5,3 & 1,56 & 1733 \\
\hline Aruba & 47,3 & 1,5 & 0,4 & 18,4 & 30,1 & 2,3 & & 1105 \\
\hline Fort de France & 76,6 & & & 8,3 & 0,7 & 14,4 & & 749 \\
\hline Grand Cayman Is. & 18,4 & 2,4 & & & 79,2 & & & 375 \\
\hline San Juan (PR) & 50,6 & 1,5 & & 0,7 & 46,8 & 0,3 & & 4164 \\
\hline Cali & & 45,1 & & 15,0 & 28,2 & 11,7 & & 206 \\
\hline Córdoba & & 17,6 & 60,2 & 17,6 & & 4,5 & & 176 \\
\hline Guadalajara & & 1,7 & & & 98,3 & & & 1058 \\
\hline Guayaquil & & 19,5 & 9,2 & 34,2 & 25,4 & 11,8 & & 524 \\
\hline Medellin & 2,8 & 45,6 & & 20,5 & 28,7 & 2,4 & & 327 \\
\hline Monterrey & & & & & 100,0 & & & 517 \\
\hline Porto Alegre & & & 93,7 & 6,3 & & & & 206 \\
\hline Recife & & & & & 41,9 & 58,1 & & 74 \\
\hline Cancún & 2,3 & 6,9 & & 0,8 & 80,1 & 9,9 & & 2201 \\
\hline Montego Bay & 1,6 & & & & 91,1 & 7,2 & & 788 \\
\hline Punta Cana & 12,1 & 10,2 & 1,2 & 4,0 & 54,0 & 18,5 & & 782 \\
\hline San Jose Cabo & & & & & 100,0 & & & 694 \\
\hline
\end{tabular}

Fuente: Elaboración propia para esta investigación a partir la base de Datos, COMBER International, 2011. 
Tan valioso como el análisis del conjunto latinoamericano resulta el comportamiento diferencial de sus ciudades en cuanto que es, sin duda, indicativo del papel que juegan en el desenclave internacional de los Estados a los que pertenecen. Desde un punto de vista general, valorando sólo conexiones y frecuencias absolutas, hay seis ciudades fundamentales en el sistema aéreo latinoamericano: Cancún, Ciudad de Panamá, Sao Paulo, Ciudad de México, Juan de Puerto Rico y Buenos Aires. No obstante, interesa valorar junto con las cifras absolutas, la direccionalidad de sus vuelos como indicador de su conexión con el sistema aéreo global. Para el estudio de estas ciudades y otras con un rol significativo dentro de sus países, se han realizado cinco grupos, en relación con los comportamientos diferenciales identificados en el epígrafe anterior.

1.- Metrópolis latinoamericanas: Ciudad de México, Sao Paulo y Buenos Aires presentan un alto número de conexiones y frecuencias y una importante diversidad en cuanto a la direccionalidad de la conexión con importantes lazos, no ya solo con los contextos cercanos y América del Norte, sino con un significativo número de ciudades europeas, y en casos excepcionales con el resto del Mundo. En este sentido, Sao Paulo se configura como la cabeza del sistema latinoamericano con conexiones con África (Johannesburgo, Ciudad del Cabo, Luanda) y Oriente Medio (Doha, Dubai y Tel Aviv). La conectividad de Ciudad de México, se ve mediatizada por la cercanía de los hubs estadounidenses, lo que se refleja en la polarización de sus flujos, aunque a favor de su rol como metrópoli continental hay que valorar que sus conexiones con Europa son similares, por ejemplo, a las de Buenos Aires. Aunque en un principio Río de Janeiro se ha incluido en este grupo, el número absoluto de conexiones y frecuencias muestra que está un escalón por debajo de las anteriores, aunque presente diferencias importantes con respecto a las ciudades medias brasileñas. Se trata, sin duda, de una ciudad con una conectividad aérea internacional que responde a la actividad turística y no solo a su rol como metrópoli latinoamericana de orden internacional.

2.- Capitales de Estado. Los valores de la varianza en relación con la distribución de los datos del cuadro 7 (cuadro 8) subrayan que las capitales nacionales tienen un comportamiento similar al de algunas metrópolis latinoamericanas (Sao Paulo y Ciudad de México, por ejemplo). Lima, Ciudad de Panamá, La Habana, Caracas y Bogotá muestran una diversidad de conexiones mayor que La Paz, Quito y San José, en las que predominan los flujos aéreos de carácter regional. Por su parte, Brasilia es un caso atípico en cuanto que no presenta conexiones con el interior de la región a la que pertenece (Cono Sur) aspecto que evidencia su creciente rol como tercera ciudad Brasil, con una proyección internacional creciente. Por el contrario, en La Paz predominan las conexiones de carácter más regional, mucho más polarizadas que en los casos anteriores, lo que nos habla de una proyección mundial inferior a otras capitales nacionales. A parte de estos aspectos generales es interesante valorar cómo la direccionalidad en la conexión de La Habana y Caracas difiere sustantivamente del resto, estando escasamente polarizada por Norteamérica, y presentado conexiones extracon- 
tinentales con Asia y África, que permiten intuir la peculiar acción política internacional que desarrollan ambos Estados.

Cuadro 8. Varianza de las conexiones y vuelos. 2011.

\begin{tabular}{|l|r|r|l|r|r|}
\hline & CONEX. & VUELOS & & CONEX. & VUELOS \\
\hline Buenos Aires & 60,5 & 336,7 & Fort de France & y & 476,4 \\
\hline Cd. de México & 251,5 & 370,9 & Grand Cayman & 390,1 & 518,9 \\
\hline Río de Janeiro & 146,5 & 185,2 & San Juan (PR) & 305,2 & 349,8 \\
\hline Sao Paulo & 106,1 & 142,0 & Cali & 171,9 & 207,6 \\
\hline Bogotá & 84,9 & 127,4 & Córdoba & 269,9 & 305,4 \\
\hline Brasilia & 171,9 & 236,5 & Guadalajara & 705,9 & 796,0 \\
\hline Caracas & 84,3 & 84,8 & Guayaquil & 127,1 & 136,8 \\
\hline Habana & 98,2 & 208,3 & Medellín & 130,6 & 220,6 \\
\hline La Paz (BO) & 371,9 & 378,1 & Monterrey & 826,4 & 826,4 \\
\hline Lima & 113,3 & 140,1 & Porto Alegre & 535,5 & 718,9 \\
\hline Ciudad de Panamá & 98,3 & 119,6 & Recife & 485,5 & 383,9 \\
\hline Quito & 244,6 & 355,3 & Cancún & 322,9 & 515,1 \\
\hline San José & 193,0 & 258,4 & Montego Bay & 384,3 & 677,1 \\
\hline Santiago (CL) & 94,4 & 251,6 & Punta Cana & 189,3 & 237,8 \\
\hline Aruba & 165,5 & 234,9 & San José Cabo & 826,4 & 826,4 \\
\hline
\end{tabular}

Fuente: Elaboración propia a partir de los datos del cuadro 7.

3.- Las capitales del Caribe tienen también un comportamiento considerablemente polarizado hacia su región de origen y hacia Norteamérica. Entre ellas destaca San Juan, cabeza del sistema caribeño, que presenta el mayor volumen de frecuencias del sistema, aunque deba ser interpretado a la luz de la conectividad pseudo-internacional con los Estados Unidos y del necesario desenclave en el contexto insular caribeño, donde el transporte aéreo juega un papel sustancial abasteciendo la oferta turística y favoreciendo la actividad financiera internacional en relación con el asentamiento de centros financieros off shore.

4.- Se han seleccionado un total de ocho ciudades medias latinoamericanas para analizar su comportamiento diferencial en relación con las metrópolis estatales. Los resultados muestran que la polarización y el alcance de éstas está muy marcado en el caso de Guadalajara y Monterrey con Norteamérica y de Portoalegre con el Cono Sur. Recife, por su parte, actúa como nodo de enlace del sistema brasileño hacia el exterior. Por el contrario, Cali, Medellín y Guayaquil muestran una direccionalidad variada que favorece la proyección hacia el exterior de sus hinterland regionales, al tiempo que compiten con las capitales de sus respectivos países; son sin duda, ejemplos de sistemas bi ó policéfalos que se perpetúan. 
5.- Para terminar este breve pero necesario repaso a la conectividad global de las ciudades latinoamericanas es necesario ahondar en la conectividad de las ciudades turísticas habida cuenta de su irrupción y del nuevo papel que cumplen en el sistema urbano, ya identificado en los apartados anteriores. Para ello se han seleccionado cuatro de las más importantes en términos de conectividad, Cancún, Montego Bay, Punta Cana y San José del Cabo. El aeropuerto de Cancún se ha configurado como el más importante de Latinoamérica y Caribe en términos de conexiones y el quinto por lo que respecta a las frecuencias. Aunque la mayor parte de la conectividad se dirija hacia Norteamérica, el interesante abanico de conexiones con Europa responde a su carácter de destino turístico global. Los tres destinos restantes presentan también una conectividad superior a algunas capitales nacionales y a gran parte de las ciudades de orden regional. Tanto Montego Bay como Punta Cana presentan una importante conectividad hacia Europa, evidencia también de la significativa demanda que procede de este continente, mientras que la polarización de la dirección que ejerce Norteamérica sobre San José del Cabo lo hace extremadamente dependiente de este mercado, lo que sin duda no favorece la consolidación del destino especialmente en épocas de crisis.

\section{CONCLUSIONES}

En las líneas anteriores se ha realizado un análisis de la trayectoria demográfica del sistema urbano latinoamericano, indagando además sobre sus relaciones con el transporte aéreo, lo que ha puesto de manifiesto la irrupción de núcleos con nuevos roles al tiempo que se han perpetuado otros ya consolidados. El artículo incide en que estas ciudades necesitan adaptar su conectividad a los nuevos tiempos de la globalización y sin duda, esta falta de adaptación impide a los territorios que rigen, y en los que se localizan, una inserción adecuada en los circuitos globales.

En primer lugar, el análisis individualizado de la evolución demográfica del sistema urbano latinoamericano ha desvelado una estructura territorial diferencialmente consolidada dependiendo de países y regiones, pero con una tendencia clara hacia la urbanización en el que son fácilmente identificables una serie de nodos que irrumpen con fuerza en relación con funciones específicas, algunas de carácter histórico, como la extracción de recursos o los frentes de colonización y otras más novedosas como es el turismo. En este sentido ha de señalarse que aunque algunas de las funciones sean tradicionales, lo novedoso es que se asientan sobre localizaciones antes pequeñas o inexistentes, lo que implica una reorganización de la red de comunicaciones, donde el transporte aéreo juega siempre un rol pionero.

En relación con estos resultados, el apartado 3 de nuestro estudio apunta las importantes las relaciones entre los sistemas urbanos y la red de transporte aéreo, tanto a escala continental como estatal, existiendo una deficiencia en la correspondencia jerárquica entre el rango que tienen los nodos por su población y el que ostentan los aeropuertos, lo que sin duda puede ser interpretado, en primera instancia, como un signo más de la polarización y excesiva centralidad en la que se organizan las redes estatales. El transporte aéreo está cumpliendo, por tanto, otras funciones, puesto que 
es posible identificar un amplio número de nodos donde la conectividad supera con creces el volumen y el rango demográfico que le correspondería. En este sentido cabe destacar, si se exceptúa Brasil, la escasa relevancia de los aeropuertos regionales, aunque sea posible identificar cierta competencia entre éstos en el interior de Estados con sistemas urbanos bi o tricéfalos (Ecuador, Colombia, Bolivia) y la emergencia en México de núcleos con una conectividad importante, evidencia de su cada vez mayor protagonismo demográfico y económico en los contextos nacional y regional como ocurre con Guadalajara, pero sobre todo con los núcleos del norte (Monterrey, Chihuahua, Tijuana y Ciudad Juárez).

La investigación también revela una doble trayectoria. Por un lado se descubre un sistema aéreo muy próximo, en términos de conectividad, al que ya existía en 1970, reflejo del escaso protagonismo de las ciudades medias. Por otro lado se observa el creciente rol que adquieren las localizaciones turísticas, que, a nuestro juicio son uno de los agentes de cambio fundamental en el sistema. En éste se ha identificado la irrupción de enclaves turísticos de orden internacional (Cancún, Puerto Plata, Punta Cana, Montego Bay, Los Cabos) pero también la de otros que sorprenden por la magnitud en que su conectividad se manifiesta como Cuzco, Porlamar, Puerto Vallarta, Foz de Iguacu e incluso Ushuaia, donde a la demanda internacional se une también la doméstica.

Un tercer aspecto que el presente estudio ha analizado es el nivel de conexión de las ciudades latinoamericanas en el sistema global, utilizando como indicador el transporte aéreo. En este sentido ha de señalarse el escaso protagonismo que tienen la casi totalidad de los nodos, pese a que algunos de ellos como Sao Paulo y Ciudad de México pertenecen a potencias económicas emergentes. La polarización, casi extrema en algunos casos, de conexiones y frecuencias nos indica claramente una inserción en los circuitos globales muy mediatizada por la componente Norte- Sur. En este sentido es importante resaltar cómo Sao Paulo juega un rol fundamental como salida del sistema aéreo latinoamericano hacia los circuitos globales, completado por Ciudad de México y Buenos Aires, adquiriendo también un importante protagonismo regional San Juan de Puerto Rico, para el desenclave del Caribe, y Ciudad de Panamá como hub macrorregional.

Por último creemos necesario destacar que los resultados obtenidos reconocen la hipótesis formulada en la introducción de este artículo, en el sentido de que la incidencia de las dinámicas territoriales globales se deben valorar en relación con las características y procesos de los territorios concretos en un contexto de competitividad global. Sólo en casos excepcionales, como Sao Paulo, Buenos Aires y Ciudad de México, se fractura ligeramente la dinámica de movilidad Norte-Sur, predominante en el escenario latinoamericano. Parece, también, que en esta región del mundo los nodos se encuentran enclavados de forma poderosa, estando las ciudades muy integradas en la realidad territorial a la que pertenecen, no verificándose en consecuencia las tesis apuntadas por S. Sassen $(1991,2001)$, respecto a la capacidad que tienen las ciudades de orden global de saltar sus hinterland regionales o nacionales para insertarse en los circuitos globales; más bien se debe afirmar que son pocas las ciudades latinoamericanas que pueden recibir el calificativo de "globales" y que aquellas con 
cierta capacidad para serlo, lo hacen en un grado moderado. Puede apuntarse además que los procesos que concurren en esta región están más cerca de las tesis de Smith (op. cit. 2003) y que, al menos, en algunas ciudades de la cúspide de la jerarquía urbana de América Latina se entrecruzan dominios escalares, experiencias globales y locales en donde el transporte aéreo puede tener un papel importante. En cualquier caso, los resultados demuestran que, para esta región, el turismo y el papel que desempeñan ciertos territorios como "paraísos turísticos y fiscales", son dos de los vectores sobresalientes de su integración en el escenario global, algo que ya señaló el geógrafo francés O. Dollfus (1990) hace ya más de dos décadas.

\section{REFERENCIAS BIBLIOGRÁFICAS}

AGUILAR A. G. Y RODRÍGUEZ, F. (1995). The dispersal of urban growth in Mexico, 1979-1990. Regional Development Studies, vol. 1, Nagoya, United Nations Centre for Regional Development.

AGUILAR A. G. Y VÁZQUEZ, M. I. (2000). Crecimiento urbano y especialización económica en México. Una caracterización regional de las funciones dominantes. Investigaciones Geográficas, $n^{\circ}$. 42, 87- 108.

AGUILAR, A. G. (2002). Las mega-ciudades y las periferias expandidas. Ampliando el concepto en Ciudad de México. Eure, Vol. 28, 85, 121-149.

AGUILAR, A. G. Y WARD C. B. (2003). Globalization, regional development, and mega-city expansion in Latin America: Analyzing Mexico City's peri-urban Hinterland. Cities, Vol. 20 (1), 3- 21.

ARIZA, M. (2003). La urbanización en México en el último cuarto del Siglo XX. Montevideo, Center for the Study of Urbanization and Internal Migration in Developing Countries (Austin).

CEPAL (2012). Estudio económico de América Latina y el Caribe: Las políticas ante las adversidades de la economía internacional. Santiago de Chile, Naciones Unidas-CEPAL.

CHION, M. (2002). Dimensión metropolitana de la globalización: Lima a fines del siglo XX. Eure, Vol. 28, 85, 71- 87.

CÓRDOBA, J. Y GAGO, C. (2002). Madrid en el escenario de un sistema mundial de ciudades. Anales de Geografía de la Universidad Complutense, Vol. extraordinario, 2002, 203- 219.

CÓRDOBA, J. Y GAGO, C. (2010). Latin American Cities and Globalisation: Change and Permanency in the Context of Development Expectations. Urban Studies, 47(9), 2003- 2021.

CÓRDOBA, J., GAGO, C. Y SERRANO, M. (2007). Transporte aéreo y espacilidad diferencial. En Gutiérrez, S. y Sanz, J. J. (Eds.): Homenaje al Profesor José Manuel Casas Torres. Madrid, UCM, 45- 64.

DEDECCA, C. S. Y BALTAR, P. E. A. (1999). Mercado de Trabalho e Informalidade nos anos 90. IE/UNICAMP (mimeo). 
DERUDDER, B. et Al. (2003). Hierarchical tendencies and regional patterns in the world city network: a global urban analysis of 234 cities. Regional Studies, 37(9), 875- 886.

DERUDDER, B. Y WILTOX, F. (2005). An appraisal of the use of airline data in assessments of the world city network. Urban Studies, 42(13). 2371- 2388.

DÍAZ ORUETA, F. Y LUNGO, M. (1999). Urbanización y Estructura Social en América Latina. En d'Entremont y Pérez Adán (eds.): Desarrollo Socioeconómico y Evolución Demográfica, Perspectivas para América Latina, Pamplona, EUNSA, 55- 70.

DOLLFUS, O. (1990). Le système monde. En Brunet, R. y Dollfus, O., Mondes Nouveaux. Paris, Hachette, 273- 529.

FERNANDES A. C. Y NEGREIROS, R. (2001). Economic developmentism and change within the Brazilian urban system. Geoforum, 32, 415- 435.

FRIEDMANN, J. (1986). The world city hypothesis. Development and Change, 17, 69-83.

FRIEDMANN, J. (1995). Where we stand: A decade or world city research. En Knox, P. L. y Taylor, P. J., (Eds.): World Cities in a World System. Cambridge, Cambridge University Press, 436-457.

GAGO, C. (2003). Región, Política y Transporte aéreo. Madrid, UCM.

GARZA, C. (2000). Ambitos de Expansión Territorial. En Garza, G. (Ed.): La Ciudad de México en el fin del segundo milenio. México. El Colegio de México y Gobierno del Distrito Federal, 237- 246.

GODFREY, B. J. Y BROWDER, J. O. (1996). Desarticulated Urbanization in Brazilian Amazon. The Geographical Review, 86 (3), 443- 445.

GUTIÉRREZ DE MCGREGOR, M. T. y González, J. (2004). Dinámica y distribución espacial de la población urbana en México, 1970-2000. México D.F., Instituto de Geografía, UNAM.

GWYNNE, R. N. (1999). Globalization, Neoliberalism and Economic Changes in South America and Mexico. En Gwynne y Kay (Eds.): Latin America Transformed: globalization and modernity. Londres, Arnold.

MASSEY, D. (1994). Space, Place and Gender. Cambridge, Polity Press.

MATA D. et Al. (2007). Determinants of city growth in Brazil. Journal of Urban Economics, 62, 252- 272.

ONU (2010). World Population Prospects. The 2010 revision. Nueva York, Naciones Unidas.

ONU- HÁBITAT (2012). Estado de las Ciudades de América Latina y Caribe. Rumbo a una nueva transición urbana. Nairobi, Programa de las Naciones Unidas para los Asentamientos Humanos.

ORTIZ, I. M. et Al. (2003). Escenarios demográficos en América Latina y el Caribe. Investigaciones Geográficas, $\mathrm{n}^{\mathrm{o}}$ 51, 107- 124.

PINTO DA CUNHA, J. M. (2003). Urbanización, redistribución espacial de la población y transformaciones socioeconómicas en América Latina. Santiago de Chile, CEPAL. 
PORTES, A. (1989). Latin American Urbanization during the Years of the Crisis. Latin American Research Review, Vol. 24, No. 3, 7- 44.

PREALC (1992). Empleo y transformación productiva en América Latina y el Caribe. Santiago de Chile, PREALC.

RYDERM R. Y BROWN, L. A. (2000). Urban-system evolution on the frontier of the Ecuadorian Amazon. The Geographical Review, 90 (4), 511- 535.

SÁNCHEZ-CRISPÍN, A. Y PROPÍN, E. (2001). Cambios en la orientación funcional de las ciudades medias del trópico mexicano. Cuadernos Geográficos, 31, pp. 6985.

SASSEN, S. (1991; 2001). The Global City: New York, London, Tokyo. Princeton, Princeton University Press.

SASSONE, S. M. (2000). Reestructuración territorial y ciudad intermedias en Argentina. Ciudad y Territorio. Estudios Territoriales, XXXII (123), 57- 92.

SMITH, D. A. Y TIMBERLAKE, M. (2001). World city networks and hierarchies, 1977-1997: an empirical analysis of global air travel links. American Behavioral Scientist, 44(10), 1656- 1678.

SMITH, R. (2003). World city actor-networks. Progress in Human Geography, 27 (1), $25-44$.

TAYLOR, P. J. (1997). Hierarchical tendencies amongst world cities: a global research proposal. Cities: The International Journal of Urban Policy and Planning, 14, 323- 332.

TAYLOR, P. J. (2000). World cities and territorial states under conditions of contemporary globalization. Political Geography, 19, 5- 32. 